MATHEMATICS OF COMPUTATION

Volume 73 , Number 246 , Pages $569-593$

S $0025-5718(03) 01552-7$

Article electronically published on May 21, 2003

\title{
THE LOCAL DISCONTINUOUS GALERKIN METHOD FOR THE OSEEN EQUATIONS
}

\author{
BERNARDO COCKBURN, GUIDO KANSCHAT, AND DOMINIK SCHÖTZAU
}

\begin{abstract}
We introduce and analyze the local discontinuous Galerkin method for the Oseen equations of incompressible fluid flow. For a class of shaperegular meshes with hanging nodes, we derive optimal a priori estimates for the errors in the velocity and the pressure in $L^{2}$ - and negative-order norms. Numerical experiments are presented which verify these theoretical results and show that the method performs well for a wide range of Reynolds numbers.
\end{abstract}

\section{INTRODUCTION}

In this paper, we introduce the local discontinuous Galerkin (LDG) method for the Oseen equations of incompressible fluid flow. We conduct stability and a priori error analysis and perform numerical tests of the scheme. The Oseen equations are

$$
\begin{aligned}
-\nu \Delta \mathbf{u}+(\beta \cdot \nabla) \mathbf{u}+\gamma \mathbf{u}+\nabla p & =\mathbf{f} & & \text { in } \Omega, \\
\nabla \cdot \mathbf{u} & =0 & & \text { in } \Omega, \\
\mathbf{u} & =\mathbf{g} & & \text { on } \Gamma,
\end{aligned}
$$

where $\mathbf{u}$ is the velocity, $p$ the pressure, $\mathbf{f} \in L^{2}(\Omega)^{d}$ a prescribed external body force, $\nu$ the kinematic viscosity, $\beta$ a convective velocity field and $\gamma$ a given scalar function. As usual, we take $\Omega$ to be a bounded domain of $\mathbb{R}^{d}, d=2,3$, with boundary $\Gamma=\partial \Omega$, and the Dirichlet data $\mathbf{g} \in H^{1 / 2}(\Gamma)^{d}$ to satisfy the compatibility condition $\int_{\Gamma} \mathbf{g} \cdot \mathbf{n} d s=0$, where $\mathbf{n}$ denotes the outward unit normal vector to $\Gamma$.

Our long-term goal is studying the LDG method for both the transient and the stationary incompressible Navier-Stokes equations, that is, for the case in which $\beta=\mathbf{u}$ in the above equations. Thus, it is reasonable to study first the Oseen equations since they appear by linearization of the incompressible Navier-Stokes equations, e.g., in Picard's iteration. Indeed, a typical situation is the one in which one has that $\mathbf{u}$ is the velocity at the current time, $\beta$ is the velocity at the previous time step and $\gamma=1 / \Delta t$; it is thus quite natural to assume that $\gamma>0$. However,

Received by the editor February 14, 2002 and, in revised form, August 21, 2002.

2000 Mathematics Subject Classification. Primary 65N30.

Key words and phrases. Finite elements, discontinuous Galerkin methods, Oseen equations.

This work was carried out while the third author was a Dunham Jackson Assistant Professor at the School of Mathematics, University of Minnesota.

The first and third authors were supported in part by the National Science Foundation (Grant DMS-0107609) and by the University of Minnesota Supercomputing Institute.

The second author was supported in part by "Deutsche Forschungsgemeinschaft" through SFB 359 and Schwerpunktprogramm ANumE). 
for the steady-state Navier-Stokes problem, we do have $\gamma \equiv 0$; for this reason, we are not going to make the assumption $\gamma>0$. Instead, we assume that

$$
\gamma(\mathbf{x})-\frac{1}{2} \nabla \cdot \beta(\mathbf{x})=: \gamma_{0}(\mathbf{x}) \geq 0, \quad \mathbf{x} \in \Omega .
$$

This condition guarantees (see, for example, [7 15]) existence and uniqueness of a solution $(\mathbf{u}, p) \in H_{g}^{1}(\Omega)^{d} \times L_{0}^{2}(\Omega)$ where, as usual,

$$
H_{g}^{1}(\Omega)^{d}:=\left\{\mathbf{u} \in H^{1}(\Omega)^{d}:\left.\mathbf{u}\right|_{\Gamma}=\mathbf{g}\right\}, \quad L_{0}^{2}(\Omega):=\left\{p \in L^{2}(\Omega): \int_{\Omega} p d \mathbf{x}=0\right\} .
$$

Therefore, this paper extends the results recently obtained by Cockburn, Kanschat, Schötzau and Schwab [12] for the LDG method for the Stokes system, that is, for the case in which $\beta=\mathbf{0}$ and $\gamma=0$.

Several arguments for the use of the LDG method for the Stokes problem have been discussed in [12. Here, let us briefly mention that since the LDG method is a natural extension of the so-called Runge-Kutta DG methods which are robust and high-order accurate methods for nonlinear hyperbolic systems (see the recent review by Cockburn and Shu [14] and the references therein), it is reasonable to expect that for problems in which convection might play an important role, like Oseen and Navier-Stokes problems, the LDG method should provide high-quality approximations for a significantly wide range of the Reynolds number,

$$
R e=\frac{L|\bar{\beta}|}{\nu},
$$

where $L$ denotes a typical length scale and $\bar{\beta}$ a typical velocity. In fact, the main objective of this paper is to show that this is indeed the case for the Oseen system (1.1).

In this paper, we also present results that are new for the Stokes system. Thus, we show how to define the parameters associated with the numerical fluxes of the LDG method such that, when $\beta=\mathbf{0}$, the condition number of the Schur complement matrix for the pressure is independent of the kinematic viscosity $\nu$; and we present new negative-order norm estimates for both the velocity and the pressure.

To give the reader an idea of the main features of the LDG method, let us compare it with other discontinuous Galerkin methods that have been devised for Oseen equations or related problems.

- In 1998, Karakashian and Jureidini 18] devised a numerical method for the stationary incompressible Navier-Stokes equations (see also the earlier work by Baker, Jureidini and Karakashian [4 on the Stokes problem). It uses a totally discontinuous approximation for the velocity which is pointwise divergence-free inside each of the elements, and a continuous approximation for the pressure. In particular, they prove that if polynomials of degree $k$ are used for the velocity and polynomials of degree $k-1$ for the pressure, the optimal order of convergence of $k$ is achieved in a suitable mesh-dependent norm which can be thought to be associated with the $H^{1}$-norm of the velocity plus the $L^{2}$-norm of the pressure. There are three main differences between the method proposed by Karakashian and Jureidini [18] and the LDG method proposed in this paper. The first is that, although both methods weakly impose the incompressibility condition, Karakashian and Jureidini use a piecewise divergence-free velocity approximation. The second is that they use continuous approximations for the pressure whereas the LDG uses a discontinuous 
approximation. In the spirit of Taylor-Hood elements, they use a triangulation associated with the velocity which is a refinement of that associated with the pressure whereas the LDG method uses the same mesh for both the velocity and the pressure. The third difference is that the method used by Karakashian and Jureidini [18] can be associated with the classical interior (IP) penalty methods developed in the late seventies (see, in particular, the paper by Baker [3]) and hence has a penalty parameter that has to be estimated properly in order to enforce the stability of the method. In contrast, the LDG method is always stable, regardless of the size of the penalty parameter. Concerning this point, it is worth noting that a computational comparison of these (and other) DG methods for a purely elliptic model problem was recently performed by Castillo [8]. In particular, he showed that for sufficiently large penalty parameters, the quality of the approximate solutions given by these methods is quite similar, but that the LDG method behaves extremely well in the range of penalty parameters for which the IP methods are not stable; this range is very difficult to find.

- Methods closely related to our approach are the mixed DG schemes introduced recently by Hansbo and Larson [16] and Toselli [28] in the context of linear elasticity and Stokes flow; these methods also use completely discontinuous approximations for the velocity and the pressure and, as in the approaches of Baker, Karakashian and Jureidini [4, 18, are based on interior penalty techniques to weakly enforce the continuity requirements on the velocity fields. In particular, if polynomials of degree $k$ are used for the velocity and polynomials of degree $k-1$ for the pressure, a standard inf-sup condition was proved by Hansbo and Larson in [16 in a broken $H^{1}$-seminorm for the velocity and the $L^{2}$-norm for the pressure, giving rise to optimal error estimates. A more refined analysis was then given by Toselli in [28], also covering hanging nodes and extensions to the $h p$-version. These results apply immediately to the LDG method considered here and in [12]. Conversely, the results in this paper for the extension of the LDG method to the Oseen equations immediately carry over to the methods of Hansbo and Larson [16] and Toselli [28.

- Another DG method is the one introduced in 1999 by Baumann and Oden [6] for the Euler and the incompressible Navier-Stokes equations; see also the application of the method to purely diffusive problems in 24, an analysis of the one-dimensional case in 2, an application to convection-diffusion problems in 5 and a review in 25. The method of Baumann and Oden, applied to a purely diffusive problem, produces a stiffness matrix with more sparsity than the stiffness matrix of the LDG method; for example, Castillo [8] showed that, in the two-dimensional case, the LDG method has about 2.5 times more nonzero entries. On the other hand, unlike the LDG method, the method of Baumann and Oden is unstable for polynomials of degree 1, produces a stiffness matrix that is not symmetric, even for self-adjoint elliptic problems, and gives sub-optimal orders of convergence in the $L^{2}$-norm. A unified analysis of the several DG methods proposed for solving second-order elliptic problems has been recently proposed by Arnold, Brezzi, Cockburn and Marini [1]. Many of the elements of their analysis can be easily extended to other problems like the one under consideration.

- In 2000, Liu and Shu 21,22 developed a numerical method using discontinuous approximations for the two-dimensional incompressible Navier-Stokes equations in a vorticity-stream function formulation; they used an LDG discretization for the vorticity equation, including the viscous terms, a standard Poisson solver using 
continuous finite elements for the stream-function, and a total variation diminishing Runge-Kutta time discretization. In [21], a proof of the $L^{2}$-stability of both the vorticity and the velocity was given, which does not depend on the regularity of the exact solutions. This property was used later in [23] to show that the method converges for the case of a vortex sheet initial data having only positive vorticity.

Now, let us briefly describe our results. We show that if we use polynomials of degree $k$ to approximate the velocity $\mathbf{u}$ and polynomials of degree $k-1$ for the pressure $p$ and the variable $\underline{\sigma}=\nu \nabla \mathbf{u}$ (we will refer to this variable as "stress" 1 ), the $L^{2}$-norm of the error in the velocity is of order $k+1$, and the $L^{2}$-norms of the errors in $p$ and $\sigma$ are of order $k$. We also prove that the error in the $H^{-\ell}$-norm of the velocity as well as the error in the $H^{-\ell-1}$-norm of the pressure are of order $\ell+k+1$ for $0 \leq \ell \leq k-1$. We point out that these rates remain invariant if the pressure $p$ and "stresses" $\underline{\sigma}$ are approximated by polynomials of degree $k$ as well.

Finally, let us stress several of the salient features of the techniques used to prove our theoretical results:

- Since our analysis of the Oseen problem is closely related to that of the Stokes system performed in [12], we are using the same notation and the same approach. For this reason, we have resisted the temptation of rewriting our error analysis in terms of the lifting operators that proved to be so useful in the unified analysis of DG methods for elliptic problems carried out in [1]. Instead, we present the analysis in a new and simpler way which emphasizes the fact that only a few upper bounds on the bilinear forms of the LDG method are needed.

- Also, it is not difficult to present a unified analysis of DG methods for the Oseen equations by combining the results presented in this paper with the approach developed in [1] for second-order elliptic problems. We have not done that in this paper in order to emphasize the treatment of the difficulties introduced by the presence of the convective velocity field $\beta$. Because of that, we have taken great care to express all the estimates in terms of dimensionless constants; this was not carried out in [12, where the kinematic viscosity was taken to be equal to 1 and the velocity $\beta$ equal to zero.

- The technique used to treat the terms of the Stokes system is exactly the one used in 12 . However, to deal with the convective term, we are not using the classical hypothesis $\gamma_{0} \geq \gamma_{\star}>0$ (see, e.g., the recent analysis of $h p$-version discontinuous Galerkin methods for convection-diffusion equations in Houston, Schwab and Süli [17]); instead, to control the terms associated with the convection, we rely on the viscous term $-\nu \Delta \mathbf{u}$.

- The regularity estimates needed to obtain the negative-order norm error estimates are obtained by a suitable adaptation of Theorem 6.9 by Karakashian and Jureidini [18] to our case.

The paper is organized as follows. In section2, we introduce and discuss the LDG method by using the typical element-by-element flux formulation; then we rewrite the method in compact form by using the classical mixed setting that is convenient to carry out the analysis. In section 3, we state and discuss our theoretical results which we prove in section 4. In section 5, we present a series of numerical results that show that the order of convergence for the approximate solution given by

\footnotetext{
${ }^{1}$ The tensor $\underline{\sigma}$ corresponds to a stress variable in elastic models. In fact, if we chose the form of the Oseen equations with symmetric gradients, $\underline{\sigma}=\nu\left(\nabla \mathbf{u}+(\nabla \mathbf{u})^{T}\right)$ would be the deviatoric stress tensor.
} 
the theoretical results are actually sharp and that the method behaves well for a large range of values of the Reynolds number. Finally, in section 6, we end with concluding remarks. Last, but not least, in the Appendix, we consider a matter of practical importance: the dependence of the condition number of the Schur complement matrix for the pressure on the choice of the stabilization parameters in the numerical fluxes. For the case $\beta=\mathbf{0}$, which is the case in which the condition number can actually be estimated, we show how to make this choice in order to render the condition number independent of the kinematic viscosity $\nu$.

\section{The LOCAL Discontinuous GALERKIN METHOD}

In this section, we introduce the LDG discretization of the Oseen equations (1.1). This is achieved by combining the LDG discretization of the Stokes problem introduced in [12] with the classical DG discretization of the convective term.

2.1. The flux formulation of the LDG method. We use the standard notation $(\nabla \mathbf{u})_{i j}=\partial_{j} u_{i}$ and $(\nabla \cdot \underline{\sigma})_{i}=\sum_{j=1}^{d} \partial_{j} \sigma_{i j}$. We also denote by $\mathbf{u} \otimes \mathbf{v}$ the matrix whose $i j$ th component is $u_{i} v_{j}$ and write

$$
\underline{\sigma}: \underline{\tau}:=\sum_{i, j=1}^{d} \sigma_{i j} \tau_{i j}, \quad \quad \mathbf{v} \cdot \underline{\sigma} \cdot \mathbf{n}:=\sum_{i, j=1}^{d} v_{i} \sigma_{i j} n_{j}=\underline{\sigma}:(\mathbf{v} \otimes \mathbf{n})
$$

Then, we introduce the "stresses" $\underline{\sigma}=\nu \nabla \mathbf{u}$ and rewrite the Oseen problem as the following system of first-order equations:

$$
\begin{aligned}
\underline{\sigma} & =\nu \nabla \mathbf{u} & & \text { in } \Omega, \\
-\nabla \cdot \underline{\sigma}+(\beta \cdot \nabla) \mathbf{u}+\gamma \mathbf{u}+\nabla p & =\mathbf{f} & & \text { in } \Omega, \\
\nabla \cdot \mathbf{u} & =0 & & \text { in } \Omega, \\
\mathbf{u} & =\mathbf{g} & & \text { on } \Gamma .
\end{aligned}
$$

Multiplying these equations by smooth test functions $\underline{\tau}, \mathbf{v}$, and $q$, respectively, and integrating by parts over an arbitrary subset $K \subset \Omega$ with outward unit normal vector $\mathbf{n}_{K}$, we obtain

$$
\begin{gathered}
\int_{K} \underline{\sigma}: \underline{\tau} d \mathbf{x}=-\nu \int_{K} \mathbf{u} \cdot \nabla \cdot \underline{\tau} d \mathbf{x}+\nu \int_{\partial K} \mathbf{u} \cdot \underline{\tau} \cdot \mathbf{n}_{K} d s \\
\int_{K}[\underline{\sigma}: \nabla \mathbf{v}-p \nabla \cdot \mathbf{v}] d \mathbf{x}-\int_{\partial K}\left[\underline{\sigma}:\left(\mathbf{v} \otimes \mathbf{n}_{K}\right)-p \mathbf{v} \cdot \mathbf{n}_{K}\right] d s \\
+\int_{K}[\gamma \mathbf{u} \cdot \mathbf{v}-\mathbf{u} \cdot \nabla \cdot(\mathbf{v} \otimes \beta)] d \mathbf{x}+\int_{\partial K} \beta \cdot \mathbf{n}_{K} \mathbf{u} \cdot \mathbf{v} d s=\int_{K} \mathbf{f} \cdot \mathbf{v} d \mathbf{x} \\
-\int_{K} \mathbf{u} \cdot \nabla q d \mathbf{x}+\int_{\partial K} \mathbf{u} \cdot \mathbf{n}_{K} q d s=0
\end{gathered}
$$

This is the weak form of the Oseen equations used to define the LDG method. We enforce the above equations on each element $K$ of a general triangulation $\mathcal{T}$ of $\Omega$ 
which can have hanging nodes and elements of various shapes. Equations (2.1)(2.3) are well defined for functions $(\underline{\sigma}, \mathbf{u}, p)$ and $(\underline{\tau}, \mathbf{v}, q)$ in $\underline{\Sigma} \times \mathbf{V} \times Q$ where

$$
\begin{aligned}
& \underline{\Sigma}:=\left\{\underline{\sigma} \in L^{2}(\Omega)^{d^{2}} \quad:\left.\sigma_{i j}\right|_{K} \in H^{1}(K), \forall K \in \mathcal{T}, 1 \leq i, j \leq d\right\}, \\
& \mathbf{V}:=\left\{\mathbf{v} \in L^{2}(\Omega)^{d}:\left.v_{i}\right|_{K} \in H^{1}(K), \forall K \in \mathcal{T}, 1 \leq i \leq d\right\}, \\
& Q:=\left\{q \in L^{2}(\Omega): \int_{\Omega} q d \mathbf{x}=0,\left.q\right|_{K} \in H^{1}(K), \forall K \in \mathcal{T}\right\} .
\end{aligned}
$$

We wish to approximate the exact solution $(\underline{\sigma}, \mathbf{u}, p)$ with functions $\left(\underline{\sigma}_{h}, \mathbf{u}_{h}, p_{h}\right)$ in the finite element space $\underline{\Sigma}_{h} \times \mathbf{V}_{h} \times Q_{h} \subset \underline{\Sigma} \times \mathbf{V} \times Q$, where

$$
\begin{aligned}
& \underline{\Sigma}_{h}:=\left\{\underline{\sigma} \in L^{2}(\Omega)^{d^{2}}:\left.\sigma_{i j}\right|_{K} \in \mathcal{S}(K), \forall K \in \mathcal{T}, 1 \leq i, j \leq d\right\}, \\
& \mathbf{V}_{h}:=\left\{\mathbf{v} \in L^{2}(\Omega)^{d}:\left.v_{i}\right|_{K} \in \mathcal{V}(K), \forall K \in \mathcal{T}, 1 \leq i \leq d\right\}, \\
& Q_{h}:=\left\{q \in L^{2}(\Omega): \int_{\Omega} q d \mathbf{x}=0,\left.q\right|_{K} \in \mathcal{Q}(K), \forall K \in \mathcal{T}\right\},
\end{aligned}
$$

and the local finite element spaces $\mathcal{S}(K), \mathcal{V}(K)$ and $\mathcal{Q}(K)$ typically consist of polynomials.

The approximate solution $\left(\underline{\sigma}_{h}, \mathbf{u}_{h}, p_{h}\right)$ is defined by imposing that for all $K \in \mathcal{T}$, for all $(\underline{\tau}, \mathbf{v}, q) \in \mathcal{S}(K)^{d^{2}} \times \mathcal{V}(K)^{d} \times \mathcal{Q}(K)$,

$$
\begin{gathered}
\int_{K}\left[\underline{\sigma}_{h}: \nabla \mathbf{v}-p_{h} \nabla \cdot \mathbf{v}\right] d \mathbf{x}-\int_{\partial K}\left[\underline{\sigma}_{h}:\left(\mathbf{v} \otimes \mathbf{n}_{K}\right)-\widehat{p}_{h} \mathbf{v} \cdot \mathbf{n}_{K}\right] d s \\
+\int_{K}\left[\gamma \mathbf{u}_{h} \cdot \mathbf{v}-\mathbf{u}_{h} \cdot \nabla \cdot(\mathbf{v} \otimes \beta)\right] d \mathbf{x}+\int_{\partial K} \beta \cdot \mathbf{n}_{K} \widehat{\mathbf{u}}_{h}^{c} \cdot \mathbf{v} d s=\int_{K} \mathbf{f} \cdot \mathbf{v} d \mathbf{x}, \\
\quad-\int_{K} \mathbf{u}_{h} \cdot \nabla q d \mathbf{x}+\int_{\partial K} \widehat{\mathbf{u}}_{h}^{p} \cdot \mathbf{n}_{K} q d s=0 .
\end{gathered}
$$

$$
\int_{K} \underline{\sigma}_{h}: \underline{\tau} d \mathbf{x}=-\nu \int_{K} \mathbf{u}_{h} \cdot \nabla \cdot \underline{\tau} d \mathbf{x}+\nu \int_{\partial K} \widehat{\mathbf{u}}_{h}^{\sigma} \cdot \underline{\tau} \cdot \mathbf{n}_{K} d s
$$

Here, $\widehat{\mathbf{u}}_{h}^{\sigma}, \widehat{\widehat{\sigma}}_{h}, \widehat{p}_{h}, \widehat{\mathbf{u}}_{h}^{c}$ and $\widehat{\mathbf{u}}_{h}^{p}$ are the so-called numerical fluxes, which are discrete approximations to traces on the boundary of the elements. We refer to $\widehat{\mathbf{u}}_{h}^{\sigma}, \widehat{\widehat{\sigma}}_{h}$ as the diffusive fluxes, whereas $\widehat{\mathbf{u}}_{h}^{c}$ is the convective flux. Moreover, $\widehat{p}_{h}$ and $\widehat{\mathbf{u}}_{h}^{p}$ are the fluxes related to the incompressibility condition on the velocity.

2.2. Definition of numerical fluxes. First, we introduce some notation associated with traces. Let $K^{+}$and $K^{-}$be two adjacent elements of $\mathcal{T}$; let $\mathbf{x}$ be an arbitrary point of the set $e=\partial K^{+} \cap \partial K^{-}$, which is assumed to have a nonzero $(d-1)$-dimensional measure, and let $\mathbf{n}^{+}$and $\mathbf{n}^{-}$be the corresponding outward unit normal vectors at that point. Let $(\underline{\sigma}, \mathbf{u}, p)$ be an elementwise smooth function $K^{ \pm}$, and let us denote by $\left(\underline{\sigma}^{ \pm}, \mathbf{u}^{ \pm}, p^{ \pm}\right)$the traces of $(\underline{\sigma}, \mathbf{u}, p)$ on $e$ from the interior of $K^{ \pm}$. Then, we define the mean values $\{[\cdot\}$ and jumps $\llbracket \cdot \rrbracket$ at $\mathbf{x} \in e$ as

$$
\begin{aligned}
& \left\{\{p\}:=\left(p^{+}+p^{-}\right) / 2, \quad \llbracket p \rrbracket:=p^{+} \mathbf{n}^{+}+p^{-} \mathbf{n}^{-},\right. \\
& \left\{\{\mathbf{u}\}:=\left(\mathbf{u}^{+}+\mathbf{u}^{-}\right) / 2, \quad \underline{\llbracket \mathbf{u} \rrbracket}:=\mathbf{u}^{+} \otimes \mathbf{n}^{+}+\mathbf{u}^{-} \otimes \mathbf{n}^{-},\right. \\
& \left\{\{\underline{\sigma}\}:=\left(\underline{\sigma}^{+}+\underline{\sigma}^{-}\right) / 2, \quad \llbracket \underline{\sigma} \rrbracket:=\underline{\sigma}^{+} \mathbf{n}^{+}+\underline{\sigma}^{-} \mathbf{n}^{-}\right. \text {. }
\end{aligned}
$$

Note that the jumps $\llbracket p \rrbracket$ and $\llbracket \emptyset \rrbracket$ are both vectors, whereas the jump $\underline{\llbracket \mathbf{u} \rrbracket}$ is a tensor of rank two. We are now ready to define the numerical fluxes. 
The diffusive numerical fluxes. We begin by defining the diffusive fluxes, $\widehat{\widehat{\sigma}}$ and $\widehat{\mathbf{u}}$, which we pick as in [12]; this choice is in turn an extension of the fluxes for the Laplace operator considered in [9] and [11]. That is, on a face $e$ inside the domain $\Omega$, we take

$$
\widehat{\widehat{\sigma}}=\left\{\{\underline{\sigma}\}-C_{11} \underline{\llbracket \mathbf{u} \rrbracket}-\llbracket \underline{\sigma} \rrbracket \otimes \mathbf{C}_{12}, \quad \widehat{\mathbf{u}}^{\sigma}=\left\{\{\mathbf{u}\}+\underline{\llbracket \mathbf{u} \rrbracket} \cdot \mathbf{C}_{12},\right.\right.
$$

and, if $e$ lies on the boundary, we take

$$
\widehat{\widehat{\sigma}}=\underline{\sigma}-C_{11}(\mathbf{u}-\mathbf{g}) \otimes \mathbf{n}, \quad \widehat{\mathbf{u}}^{\sigma}=\mathbf{g} .
$$

The parameters $C_{11}$ and $\mathbf{C}_{12}$ depend on $\mathbf{x} \in e$ and have to be chosen properly. Their purpose is to enhance the stability and accuracy of the LDG method; see 9, 11. Note that the numerical flux $\widehat{\mathbf{u}}^{\sigma}$ is independent of the variable $\underline{\sigma}$. For this reason, it is possible to eliminate it from the equations by using the equation (2.4) to solve for $\underline{\sigma}_{h}$ in terms of $\mathbf{u}_{h}$, in an element-by-element manner; see, e.g., [9, 13 , for more details.

The numerical fluxes related to the incompressibility constraint. The numerical fluxes associated with the incompressibility constraint, $\widehat{\mathbf{u}}^{p}$ and $\widehat{p}$, are defined by using an analogous recipe; see [12. If the face $e$ is on the interior of $\Omega$, we take

$$
\widehat{\mathbf{u}}^{p}=\left\{\{\mathbf{u}\}+D_{11} \llbracket p \rrbracket+\mathbf{D}_{12} \operatorname{tr} \underline{\llbracket \mathbf{u} \rrbracket}, \quad \widehat{p}=\{\{p\}\}-\mathbf{D}_{12} \cdot \llbracket p \rrbracket,\right.
$$

where $D_{11}, \mathbf{D}_{12}$ depend on $\mathbf{x} \in e$. Here, $\operatorname{tr} \llbracket \mathbf{u} \rrbracket$ denotes the trace of $\llbracket \mathbf{u} \rrbracket$. On the boundary, we set

$$
\widehat{\mathbf{u}}^{p}=\mathbf{g}, \quad \widehat{p}=p^{+} .
$$

The convective numerical flux. For the convective flux $\widehat{\mathbf{u}}^{c}$ in (2.5), we take the standard upwind flux introduced in [20, 26], namely,

$$
\widehat{\mathbf{u}}^{c}(\mathbf{x})=\lim _{\epsilon \searrow 0} \mathbf{u}(\mathbf{x}-\epsilon \beta(\mathbf{x})) .
$$

This completes the definition of the LDG method. Next, following [12], we recast the LDG method in a classical mixed setting. We do this at this point to introduce a mesh-dependent seminorm that appears naturally in the error analysis of the method; an estimate of the error in this seminorm is a key ingredient of the analysis.

2.3. The compact formulation of the LDG method. First, we sum equations (2.4) - (2.6) over all elements, taking into account the above form of the numerical fluxes. A second integration by parts of the volume terms $\int_{K} \underline{\sigma}_{h}: \nabla \mathbf{v} d \mathbf{x}$ and $\int_{K} \mathbf{u}_{h} \cdot \nabla q d \mathbf{x}$ in (2.5) and (2.6), respectively, and elementary manipulations then show that the approximate solution $\left(\underline{\sigma}_{h}, \mathbf{u}_{h}, p_{h}\right) \in \underline{\Sigma}_{h} \times \mathbf{V}_{h} \times Q_{h}$ of the LDG method satisfies the equations

$$
\begin{array}{rr}
a\left(\underline{\sigma}_{h}, \underline{\tau}\right)+b\left(\mathbf{u}_{h}, \underline{\tau}\right) & =f(\underline{\tau}), \\
-b\left(\mathbf{v}, \underline{\sigma}_{h}\right)+c\left(\mathbf{u}_{h}, \mathbf{v}\right)+d\left(\mathbf{v}, p_{h}\right)+\mathcal{O}\left(\mathbf{u}_{h}, \mathbf{v}\right) & =g(\mathbf{v}), \\
-d\left(\mathbf{u}_{h}, q\right)+e\left(p_{h}, q\right) & =h(q),
\end{array}
$$


for all $(\underline{\tau}, \mathbf{v}, q) \in \underline{\Sigma}_{h} \times \mathbf{V}_{h} \times Q_{h}$. Here,

$$
\begin{aligned}
& a(\underline{\sigma}, \underline{\tau}):=\frac{1}{\nu} \int_{\Omega} \underline{\sigma}: \underline{\tau} d \mathbf{x}, \\
& b(\mathbf{u}, \underline{\tau}):=\sum_{K \in \mathcal{T}} \int_{K} \mathbf{u} \cdot \nabla \cdot \underline{\tau} d \mathbf{x}-\int_{\mathcal{E}_{\mathcal{I}}}\left(\{\mathbf{u}\}+\underline{\llbracket \mathbf{u} \rrbracket} \cdot \mathbf{C}_{12}\right) \cdot \llbracket \tau \rrbracket d s, \\
& c(\mathbf{u}, \mathbf{v}):=\int_{\mathcal{E}_{\mathcal{I}}} C_{11} \underline{\llbracket \mathbf{u} \rrbracket}: \underline{\llbracket \rrbracket} d s+\int_{\mathcal{E}_{\mathcal{D}}} C_{11}(\mathbf{u} \otimes \mathbf{n}):(\mathbf{v} \otimes \mathbf{n}) d s, \\
& d(\mathbf{v}, p):=-\sum_{K \in \mathcal{T}} \int_{K} p \nabla \cdot \mathbf{v} d \mathbf{x}+\int_{\mathcal{E}_{\mathcal{I}}}\left(\{[p\}]-\mathbf{D}_{12} \cdot \llbracket p \rrbracket\right) \llbracket \mathbf{v} \rrbracket d s+\int_{\mathcal{E}_{\mathcal{D}}} p \mathbf{v} \cdot \mathbf{n} d s, \\
& e(p, q):=\int_{\mathcal{E}_{\mathcal{I}}} D_{11} \llbracket p \rrbracket \cdot \llbracket q \rrbracket d s,
\end{aligned}
$$

where $\mathcal{E}_{\mathcal{I}}$ denotes the union of all interior faces of the triangulation $\mathcal{T}$ and $\mathcal{E}_{\mathcal{D}}$ the union of faces lying on $\Gamma$.

All the above bilinear forms are associated with the Stokes system; only the following bilinear form captures all the characteristic features that distinguish the Oseen equations from the Stokes equations:

$$
\mathcal{O}(\mathbf{u}, \mathbf{v}):=\sum_{K \in \mathcal{T}} \int_{K}[\gamma \mathbf{u} \cdot \mathbf{v}-\mathbf{u} \cdot \nabla \cdot(\mathbf{v} \otimes \beta)] d \mathbf{x}+\sum_{K} \int_{\partial K \backslash \Gamma_{-}} \beta \cdot \mathbf{n}_{K} \widehat{\mathbf{u}}^{c} \cdot \mathbf{v} d s,
$$

where $\Gamma_{-}=\{\mathbf{x} \in \Gamma: \beta(\mathbf{x}) \cdot \mathbf{n}<0\}$ is the so-called inflow part of the boundary $\Gamma$.

The linear forms on the right-hand side are defined as follows:

$$
\begin{aligned}
& f(\underline{\tau}):=\int_{\mathcal{E}_{\mathcal{D}}} \mathbf{g} \cdot \underline{\tau} \cdot \mathbf{n} d s \\
& g(\mathbf{v}):=\int_{\Omega} \mathbf{f} \cdot \mathbf{v} d \mathbf{x}+\int_{\mathcal{E}_{\mathcal{D}}} C_{11}(\mathbf{g} \otimes \mathbf{n}):(\mathbf{v} \otimes \mathbf{n}) d s-\int_{\Gamma_{-}} \beta \cdot \mathbf{n} \mathbf{g} \cdot \mathbf{v} d s, \\
& h(q):=-\int_{\mathcal{E}_{\mathcal{D}}} \mathbf{g} \cdot \mathbf{n} q d s .
\end{aligned}
$$

Notice that only the last term of the linear functional $g(\cdot)$ differs from the corresponding Stokes discretization.

Now, we rewrite the mixed system (2.12) in the following compact form: Find $\left(\underline{\sigma}_{h}, \mathbf{u}_{h}, p_{h}\right) \in \underline{\Sigma}_{h} \times \mathbf{V}_{h} \times Q_{h}$ such that

$$
\mathcal{A}\left(\underline{\sigma}_{h}, \mathbf{u}_{h}, p_{h} ; \underline{\tau}, \mathbf{v}, q\right)=\mathcal{F}(\underline{\tau}, \mathbf{v}, q) \quad \forall(\underline{\tau}, \mathbf{v}, q) \in \underline{\Sigma}_{h} \times \mathbf{V}_{h} \times Q_{h},
$$

where

$$
\begin{aligned}
\mathcal{A}(\underline{\sigma}, \mathbf{u}, p ; \underline{\tau}, \mathbf{v}, q):= & a(\underline{\sigma}, \underline{\tau})+b(\mathbf{u}, \underline{\tau})-b(\mathbf{v}, \underline{\sigma})+c(\mathbf{u}, \mathbf{v}) \\
& +d(\mathbf{v}, p)-d(\mathbf{u}, q)+e(p, q)+\mathcal{O}(\mathbf{u}, \mathbf{v}), \\
\mathcal{F}(\underline{\tau}, \mathbf{v}, q):= & f(\underline{\tau})+g(\mathbf{v})+h(q) .
\end{aligned}
$$

We can now introduce the seminorm which appears naturally in the error analysis of this method. It is denoted by $|\cdot|_{\mathcal{A}}$ and is defined by

$$
|(\underline{\sigma}, \mathbf{u}, p)|_{\mathcal{A}}^{2}:=\mathcal{A}(\underline{\sigma}, \mathbf{u}, p ; \underline{\sigma}, \mathbf{u}, p) .
$$

To see that $|\cdot|_{\mathcal{A}}$ is actually a seminorm, we only have to realize that we can write

$$
\mathcal{A}(\underline{\sigma}, \mathbf{u}, p ; \underline{\sigma}, \mathbf{u}, p)=\|\underline{\sigma}\|_{a}^{2}+|\mathbf{u}|_{c}^{2}+|p|_{e}^{2}+|\mathbf{u}|_{\mathcal{O}}^{2},
$$


where

$$
\begin{aligned}
& \|\underline{\sigma}\|_{a}^{2}=a(\underline{\sigma}, \underline{\sigma})=\frac{1}{\nu}\|\underline{\sigma}\|_{0, \Omega}^{2} \\
& |\mathbf{u}|_{c}^{2}=c(\mathbf{u}, \mathbf{u})=\left.\int_{\mathcal{E}_{\mathcal{I}}} C_{11}\left|\underline{\left.\underline{\llbracket \mathbf{u} \rrbracket}\right|^{2}} d s+\int_{\mathcal{E}_{\mathcal{D}}} C_{11}\right| \mathbf{u}\right|^{2} d s \\
& |p|_{e}^{2}=e(p, p)=\int_{\mathcal{E}_{\mathcal{I}}} D_{11}|\llbracket p \rrbracket|^{2} d s \\
& |\mathbf{u}|_{\mathcal{O}}^{2}=\mathcal{O}(\mathbf{u}, \mathbf{u})=\left\|\gamma_{0}^{\frac{1}{2}} \mathbf{u}\right\|_{0, \Omega}^{2}+\frac{1}{2} \int_{\mathcal{E}_{\mathcal{I}}}|\beta \cdot \mathbf{n}| \underline{|\underline{\llbracket \mathbf{u} \rrbracket}|^{2}} d s+\frac{1}{2} \int_{\Gamma}|\beta \cdot \mathbf{n} \| \mathbf{u}|^{2} d s
\end{aligned}
$$

since the kinematic viscosity $\nu$ and the stabilization parameters $C_{11}$ and $D_{11}$ are positive. The last identity is a classical result which is a direct consequence of the definition of the form $\mathcal{O}(\cdot, \cdot)$ and the assumption (1.2). Note that in the integral over $\mathcal{E}_{\mathcal{I}}, \mathbf{n}$ denotes any unit normal to the edges that belong to $\mathcal{E}_{\mathcal{I}}$.

\section{Theoretical Results}

In this section, we present and discuss our theoretical results.

3.1. The assumptions. We begin by stating our assumptions on the exact solution, the meshes, the local finite element spaces and the parameters in the definition of the numerical fluxes.

We take $\beta$ and $\gamma$ such that

$$
\beta \in L^{\infty}(\Omega)^{d}, \quad \gamma \in L^{\infty}(\Omega), \quad \gamma-\nabla \cdot \beta \in L^{\infty}(\Omega)
$$

and assume the following standard smoothness properties for the exact solution

$$
\mathbf{u} \in H^{s+1}(\Omega)^{d}, \quad p \in H^{s}(\Omega),
$$

with integer $s \geq 1$.

We assume that every element $K$ of the triangulation $\mathcal{T}$ is affinely equivalent (see 10, Section 2.3]) to one of several reference elements in an arbitrary but fixed set; this allows us to use elements of various shapes with possibly curved boundaries. For each $K \in \mathcal{T}$, we denote by $h_{K}$ the diameter of $K$ and by $\varrho_{K}$ the diameter of the largest ball included in $K$; we set, as usual, $h:=\max _{K \in \mathcal{T}} h_{K}$. The triangulations we consider have to be shape-regular, that is, there exists a positive constant $\delta_{1}$ such that (see [10, Section 3.1])

$$
\frac{h_{K}}{\varrho_{K}} \leq \delta_{1}, \quad \forall K \in \mathcal{T} .
$$

We assume that the triangulation covers the whole domain $\Omega$ and that the intersection of two grid cells is lower-dimensional. It is explicitly admitted that the intersection of two edges (in 2D) is a strict subset of both edges (surfaces in 3D). Furthermore, the maximum number of neighbors of a given element $K$ has to be bounded independently of the mesh size. This assumption is formalized by requiring that there exists a positive constant $\delta_{2}<1$ such that

$$
\delta_{2} \leq \frac{h_{K^{\prime}}}{h_{K}} \leq \delta_{2}^{-1},
$$

whenever the ( $d-1)$-dimensional Lebesgue measure of the intersection of the boundaries of the elements $K$ and $K^{\prime}$ is not zero. 
The only mild conditions we impose on the local spaces are the following:

$$
\begin{aligned}
& u \in \mathcal{V}(K): \int_{K} \nabla u \cdot \mathbf{v} d \mathbf{x}=0 \quad \forall \mathbf{v} \in \mathcal{S}^{d}(K) \quad \text { implies } \quad \nabla u \equiv \mathbf{0} \text { on } K, \\
& q \in \mathcal{Q}(K): \int_{K} \mathbf{v} \cdot \nabla q d \mathbf{x}=0 \quad \forall \mathbf{v} \in \mathcal{V}^{d}(K) \quad \text { implies } \quad \nabla q \equiv \mathbf{0} \text { on } K .
\end{aligned}
$$

These are the same conditions used in the study of LDG methods for the Stokes system in 12] they ensure that the approximate solution is well defined (this follows from [12, Proposition 2.1] and the coercivity property in (2.15)).

In order to guarantee certain approximation properties of the local spaces, we assume that they contain at least all polynomials of degree $k \geq 1$ and $k-1$, respectively, that is,

$$
P^{k}(K) \subseteq \mathcal{V}(K), \quad P^{k-1}(K) \subseteq \mathcal{S}(K), \quad P^{k-1}(K) \subseteq \mathcal{Q}(K) .
$$

Let us point out that in [12, it was shown that, for the Stokes system, the same orders of convergence are obtained if all the above local spaces are taken to be $P^{k}(K)$ and that it is not less efficient to use the equal-order spaces.

We choose the stabilization coefficients $C_{11}$ and $D_{11}$ as follows

$$
\begin{aligned}
& C_{11}(\mathbf{x})= \begin{cases}c_{11} \max \left\{h_{K}^{-1}, h_{K^{\prime}}^{-1}\right\} & \text { if } \mathbf{x} \text { in the interior of } \partial K \cup \partial K^{\prime}, \\
c_{11} h_{K}^{-1} & \text { if } \mathbf{x} \text { in the interior of } \partial K \cap \Gamma,\end{cases} \\
& D_{11}(\mathbf{x})=\left\{d_{11} \max \left\{h_{K}, h_{K^{\prime}}\right\}\right.
\end{aligned}
$$

with parameters $c_{11}$ and $d_{11}$ independent of the mesh size. A simple dimensional analysis reveals that these parameters must be taken as

$$
c_{11} \sim \nu, \quad d_{11} \sim \nu^{-1}
$$

and that $\left|\mathbf{C}_{12}\right|$ as well as $\left|\mathbf{D}_{12}\right|$ must be of order one. In particular, this choice ensures that the condition numbers of the matrices associated with the method are independent of the kinematic viscosity $\nu$; see the Appendix, where the impact of this choice on the condition number of the matrices of the method is explored.

3.2. The results. In this section, we state and discuss our main results. These are a priori error estimates of the approximation error in the so-called $\mathcal{A}$-seminorm defined in (2.14). We also present an estimate of the $L^{2}$-norm of the error in the pressure and an estimate of negative-order norms of the error in the velocity and the pressure.

To express our estimates in terms of dimensionless quantities, we use a slightly unusual definition of these norms. Namely, for any integer $\ell \geq 0$, we set

$$
\|u\|_{\ell, \Omega}=\left\{c_{i} \sum_{i=0}^{\ell}|u|_{i, \Omega}^{2}\right\}^{1 / 2}, \quad|u|_{i, \Omega}=\left\{\sum_{|\alpha|=i}\left\|D^{\alpha} u\right\|_{0, \Omega}^{2}\right\}^{1 / 2},
$$

with parameters $c_{i}>0$ independent of the discretization. These parameters solely depend on the measure units and are chosen in such a way that all the seminorms are dimensionally equivalent. The Sobolev norm $\|\cdot\|_{-\ell, \Omega}, \ell \geq 0$, is then given by

$$
\|u\|_{-\ell, \Omega}=\sup _{\phi \in \mathcal{C}_{0}^{\infty}(\Omega)} \frac{\int_{\Omega} u(x) \phi(x) d x}{\|\phi\|_{\ell, \Omega}} .
$$


To measure the pressure $p \in L_{0}^{2}(\Omega)$ in negative-order norms, we define similarly

$$
\|p\|_{-\ell, \Omega}=\sup _{\phi \in \mathcal{C}_{0}^{\infty}(\Omega) \cap L_{0}^{2}(\Omega)} \frac{\int_{\Omega} p(x) \phi(x) d x}{\|\phi\|_{\ell, \Omega}} .
$$

We are also going to use the following norm,

$$
\|(\mathbf{u}, p)\|_{s}=\nu^{\frac{1}{2}}\|\mathbf{u}\|_{s+1, \Omega}+\nu^{-\frac{1}{2}}\|p\|_{s, \Omega},
$$

for integer $s$. Note that the two terms of the above norm have the same dimensions if the parameters $c_{i}$ above are suitably chosen.

The error estimates we present next are stated in terms of the constant $\kappa$ in the continuous inf-sup condition for the divergence operator [7, 15]:

$$
\inf _{q \in L_{0}^{2}(\Omega)} \sup _{\mathbf{v} \in H_{0}^{1}(\Omega)^{d}} \frac{\int_{\Omega} q \nabla \cdot \mathbf{v} d \mathbf{x}}{\|q\|_{0, \Omega}\|\mathbf{v}\|_{1, \Omega}} \geq \kappa=\kappa(\Omega)>0,
$$

and in terms of the following two other dimensionless parameters:

$$
\begin{aligned}
\mu_{h} & =\max \left\{\frac{h\|\beta\|_{L^{\infty}(\Omega)^{d}}}{\nu}, \frac{h^{2}\|\gamma-\nabla \cdot \beta\|_{L^{\infty}(\Omega)}}{\nu}\right\}, \\
M_{h} & =\frac{h C_{\text {Poinc }}\|\gamma-\nabla \cdot \beta\|_{L^{\infty}(\Omega)}}{\nu},
\end{aligned}
$$

where $C_{\text {Poinc }}$ is the Poincaré constant that we use here to dimensionally balance the term $M_{h}$. Note that the number $\nu^{-1} h\|\beta\|_{L^{\infty}(\Omega)^{d}}=L^{-1} h$ Re is the cell Peclet number which can be thought of as being a measure of the numerical resolution of the convection. Similarly, the other parameter can be thought of as a measure of how well the "reaction" is being resolved.

Finally, we assume that the boundary of the domain $\Omega$ is of class $C^{\ell+2}$ for some $\ell \geq 0$. This ensures that the so-called dual problem is well posed in the norm $\|(\cdot, \cdot)\|_{-\ell-1}$; see [4, 18, 27].

We have the following result.

Theorem 3.1. Under the assumptions of section 3.1 and for all $h<h_{0}$, where $h_{0}$ solely depends on the data, we have that the error $\left(\underline{e}_{\sigma}, \mathbf{e}_{u}, e_{p}\right)$ between the exact solution $(\underline{\sigma}, \mathbf{u}, p)$ and the LDG approximation $\left(\underline{\sigma}_{h}, \mathbf{u}_{h}, p_{h}\right)$ satisfies the following bounds:

$$
\begin{aligned}
\left|\left(\underline{e}_{\sigma}, \mathbf{e}_{u}, e_{p}\right)\right|_{\mathcal{A}} & \leq C_{\mathcal{A}} h^{\min \{s, k\}}\|(\mathbf{u}, p)\|_{s}, \\
\nu^{-\frac{1}{2}}\left\|e_{p}\right\|_{0, \Omega} & \leq \frac{C_{p}}{\kappa} h^{\min \{s, k\}}\|(\mathbf{u}, p)\|_{s}, \\
\left\|\left(\mathbf{e}_{u}, e_{p}\right)\right\|_{-\ell-1} & \leq C_{-\ell-1} h^{\min \{\ell+1, k\}+\min \{k, s\}}\|(\mathbf{u}, p)\|_{s} \quad \forall \ell \geq 0,
\end{aligned}
$$

where the dimensionless constants $C_{\mathcal{A}}, C_{p}$ and $C_{-\ell-1}$ are continuous functions of $\mu_{h}\left(C_{p}\right.$ also depends on $\left.M_{h}\right)$ and depend only on $k$ and the mesh constants in (3.3) and (3.4).

In particular, for $\ell=0$, the bound (3.16) implies the $L^{2}$-norm error estimate

$$
\nu^{\frac{1}{2}}\left\|\mathbf{e}_{u}\right\|_{0, \Omega} \leq C_{-1} h^{\min \{1, k\}+\min \{k, s\}}\|(\mathbf{u}, p)\|_{s}
$$

of the velocity.

Let us briefly discuss this result:

- If we use polynomials of degree $k$ for the velocity $\mathbf{u}$ and polynomials of degree $k-1$ for the "stresses" $\underline{\sigma}$ and for the pressure $p$, this theorem states that we obtain 
an order of convergence of $k+1$ for the $L^{2}$-norm of the error in the velocity and of $k$ for the $L^{2}$-norm of the error in $\underline{\sigma}$ and $p$.

- Let $\Lambda(\mathbf{u}, p)$ be a linear functional such that

$$
|\Lambda(\mathbf{u}, p)| \leq C\|(\mathbf{u}, p)\|_{-\ell-1} .
$$

Then, by linearity,

$$
\left|\Lambda\left(\mathbf{e}_{u}, e_{p}\right)\right| \leq C\left\|\left(\mathbf{e}_{u}, e_{p}\right)\right\|_{-\ell-1} .
$$

This simple calculation shows that the theorem also implies that the error of smooth linear functionals of the velocity and the pressure converges with an order of $2 k$.

- If we use polynomial approximations of degree $k$ for all the unknowns, it is easy to prove that the above orders of convergence remain the same. This fact is actually verified in our numerical experiments for which we have used rectangular elements and tensor product polynomials of degree $k$. This choice of polynomial spaces is motivated by the fact that, in the case of the Stokes system investigated in [12], using equal-degree polynomial spaces has been shown to be not less efficient than using polynomials of degree $k-1$ for $\underline{\sigma}$ and $p$.

- The condition $h<h_{0}$ does not have any practical relevance since the numerical scheme is well defined for any mesh.

- The error estimates in the $\mathcal{A}$-seminorm and the $L^{2}$-error estimate for the pressure hold true for any bounded Lipschitz domain $\Omega \subset \mathbb{R}^{d}$ whereas the $L^{2}$-norm error bound (3.17) for the velocity is proved by a duality argument for smooth domains of class $C^{2}$. As can be inferred from our proof in section 4 and standard elliptic regularity results, the $L^{2}$-bound (3.17) for the velocity also holds true on two-dimensional convex polygons, cf., e.g., [15, Remark 5.6].

- It is still possible to have a well-defined LDG method if we take $D_{11} \equiv 0$. Indeed, for the mixed-order polynomial spaces $\mathcal{V}(K)=P^{k}(K), \mathcal{S}(K)=P^{k-1}(K)$ and $\mathcal{Q}(K)=P^{k-1}(K)$, we can proceed as in [16, Section 4] (see [28, Section 6.2] for tensor product polynomial spaces) to prove a standard inf-sup condition and obtain identical error estimates. However, when using the same polynomial degree $k$ for all the unknowns, this approach is no longer available and the stabilization terms induced by $D_{11}$ of the order $\mathcal{O}(h)$ seem to be necessary.

\section{Proof of Theorem 3.1}

This section is devoted to proving Theorem 3.1. To do that, we are not going to assume that the function $\gamma_{0}$ in (1.2) is strictly larger than a positive parameter. This renders the proof more delicate since we cannot control the convective term as is usually done; instead, we use the stabilizing effects of the viscosity.

To be able to do that, the numerical scheme should have captured these viscosity effects in a suitable manner. The mathematical condition that reflects this fact is

$$
C_{\text {stab }} C_{\mathcal{O}} \mu_{h}<1
$$

where $C_{\text {stab }}$ is the stability constant of the dual problem and $C_{\mathcal{O}}$ is a continuity constant of the bilinear form associated with the convective term. The condition $h<h_{0}$ in Theorem 3.1 is nothing but a rewriting of the above inequality.

We proceed in several steps. 
4.1. Step 1: Estimates of the bilinear forms. We begin with a result that contains all the estimates of the bilinear forms of the LDG method needed to carry out the error analysis.

We denote by $\underline{\Pi}, \Pi$ and $\Pi$ the $L^{2}$-projections into the finite element spaces $\underline{\Sigma}_{h}$, $\mathbf{V}_{h}$ and $Q_{h}$, respectively, and set $\underline{\xi}_{\sigma}=\underline{\sigma}-\underline{\Pi \sigma}, \xi_{u}=\mathbf{u}-\Pi \mathbf{u}$, as well as $\xi_{p}=p-\Pi p$.

Lemma 4.1. Assume that $(\mathbf{u}, p)$ belongs to $H^{s+1}(\Omega)^{d} \times H^{s}(\Omega)$ and that $(\mathbf{v}, q)$ belongs to $H^{t+1}(\Omega)^{d} \times H^{t}(\Omega)$; set $\underline{\sigma}=\nabla \mathbf{u}$ and $\underline{\tau}=\nabla \mathbf{v}$. Then we have

$$
\begin{aligned}
\left\|\underline{\xi}_{\sigma}\right\|_{a} & \leq C_{a} h^{\min \{s, k\}}\|(\mathbf{u}, 0)\|_{s}, & & \\
\left|b\left(\xi_{u}, \underline{\tau}_{h}\right)\right| & \leq C_{b} h^{\min \{s, k\}}\|(\mathbf{u}, 0)\|_{s}\left\|_{\tau_{h}}\right\|_{a}, & & \forall \underline{\tau}_{h} \in \underline{\Sigma}_{h}, \\
\left|b\left(\mathbf{v}_{h}, \underline{\xi}_{\sigma}\right)\right| & \leq C_{b} h^{\min \{s, k\}}\|(\mathbf{u}, 0)\|_{s}\left|\mathbf{v}_{h}\right|_{c}, & & \forall \mathbf{v}_{h} \in \mathbf{V}_{h}, \\
\left|b\left(\xi_{u}, \underline{\xi}_{\tau}\right)\right| & \leq C_{b}^{2} h^{\min \{s, k\}\|(\mathbf{u}, 0)\|_{s} h^{\min \{t, k\}}\|(\mathbf{v}, 0)\|_{t},} & & \\
\left|\xi_{u}\right|_{c} & \leq C_{c} h^{\min \{s, k\}\|(\mathbf{u}, 0)\|_{s},} & & \forall q_{h} \in Q_{h}, \\
\left|d\left(\xi_{u}, q_{h}\right)\right| & \leq C_{d} h^{\min \{s, k\}}\|(\mathbf{u}, 0)\|_{s}\left|q_{h}\right|_{e}, & & \forall \mathbf{v}_{h} \in \mathbf{V}_{h}, \\
\left|d\left(\mathbf{v}_{h}, \xi_{p}\right)\right| & \leq C_{d} h^{\min \{s, k\}}\|(\mathbf{0}, p)\|_{s}\left|\mathbf{v}_{h}\right|_{c}, & & \\
\left|d\left(\xi_{u}, \xi_{q}\right)\right| & \leq C_{d}^{2} h^{\min \{s, k\}}\|(\mathbf{u}, 0)\|_{s} h^{\min \{t, k\}}\|(\mathbf{0}, q)\|_{t}, & & \leq C_{e} h^{\min \{s, k\}}\|(\mathbf{0}, p)\|_{s},
\end{aligned}
$$

where the constants $C_{a}, C_{b}, C_{c}, C_{d}$, and $C_{e}$ depend solely on inverse inequality and approximation constants. Moreover,

$$
\begin{aligned}
& \left|\mathcal{O}\left(\xi_{u}, \mathbf{v}_{h}\right)\right| \leq C_{\mathcal{O}} \mu_{h} h^{\min \{s, k\}}\|(\mathbf{u}, 0)\|_{s}\left(\left|\mathbf{v}_{h}\right|_{c}+\nu^{\frac{1}{2}} h^{-1}\left\|\mathbf{v}_{h}\right\|_{0, \Omega}\right), \quad \forall \mathbf{v}_{h} \in \mathbf{V}_{h}, \\
& \left|\mathcal{O}\left(\mathbf{v}_{h}, \xi_{u}\right)\right| \leq C_{\mathcal{O}} \mu_{h} h^{\min \{s, k\}}\|(\mathbf{u}, 0)\|_{s}\left(\left|\mathbf{v}_{h}\right|_{c}+\nu^{\frac{1}{2}} h^{-1}\left\|\mathbf{v}_{h}\right\|_{0, \Omega}\right), \quad \forall \mathbf{v}_{h} \in \mathbf{V}_{h}, \\
& \left|\mathcal{O}\left(\xi_{u}, \xi_{v}\right)\right| \leq C_{\mathcal{O}}^{2} \mu_{h} h^{\min \{s, k\}}\|(\mathbf{u}, 0)\|_{s} h^{\min \{t, k\}}\|(\mathbf{v}, 0)\|_{t},
\end{aligned}
$$

where $C_{\mathcal{O}}$ depends solely on inverse inequality and approximation constants.

Finally, if $\mathbf{w} \in H_{0}^{1}(\Omega)^{d}$, then

$$
\begin{array}{ll}
|b(\mathbf{w}, \underline{\sigma})| \leq \nu^{\frac{1}{2}}|\mathbf{w}|_{1, \Omega}\|\underline{\sigma}\|_{a}, & \forall \underline{\sigma} \in \underline{\Sigma}, \\
|\mathcal{O}(\mathbf{v}, \mathbf{w})| \leq\left(M_{h}+\mu_{h}\right) \nu^{\frac{1}{2}}|\mathbf{w}|_{1, \Omega} \nu^{\frac{1}{2}} h^{-1}\|\mathbf{v}\|_{0, \Omega}, & \forall \mathbf{v} \in \mathbf{V} .
\end{array}
$$

Proof. The first set of estimates have been proved in [12; see [12 Corollaries 3.4 and 3.8]. To prove the estimates for the Oseen bilinear form, we proceed as follows. We begin by noting that we can write,

$$
\mathcal{O}\left(\xi_{u}, \mathbf{v}_{h}\right)=T_{1}+T_{2}+T_{3}
$$

where

$$
\begin{aligned}
T_{1} & =\sum_{K \in \mathcal{T}} \int_{K}(\gamma-\nabla \cdot \beta) \xi_{u} \cdot \mathbf{v}_{h} d \mathbf{x}, \\
T_{2} & =-\sum_{K \in \mathcal{T}} \int_{K} \xi_{u} \cdot(\beta \cdot \nabla) \mathbf{v}_{h} d \mathbf{x}, \\
T_{3} & =\sum_{e \subset \Gamma_{+}} \int_{e}\left(\widehat{\xi_{u}^{c}} \otimes \beta\right):\left(\mathbf{v}_{h} \otimes \mathbf{n}\right) d s+\sum_{e \subset \mathcal{E}_{\mathcal{I}}} \int_{e}\left(\widehat{\xi}_{u}^{c} \otimes \beta\right): \underline{\llbracket \mathbf{v}_{h} \rrbracket} d s,
\end{aligned}
$$


and where $\Gamma_{+}=\{\mathbf{x} \in \Gamma: \beta(\mathbf{x}) \cdot \mathbf{n} \geq 0\}$ is the outflow boundary of $\Gamma$. Using standard approximation properties of the $L^{2}$-projection, we find that

$$
\begin{aligned}
\left|T_{1}\right| & \leq \sum_{K \in \mathcal{T}}\|\gamma-\nabla \cdot \beta\|_{L^{\infty}(K)}\left\|\xi_{u}\right\|_{0, K}\left\|\mathbf{v}_{h}\right\|_{0, K} \\
& \leq C h\|\gamma-\nabla \cdot \beta\|_{L^{\infty}(\Omega)} h^{\min \{s, k\}}|u|_{s+1, \Omega}\left\|\mathbf{v}_{h}\right\|_{0, \Omega}, \\
& \leq C \mu_{h} h^{\min \{s, k\}} \nu^{\frac{1}{2}}|u|_{s+1, \Omega} \nu^{\frac{1}{2}} h^{-1}\left\|\mathbf{v}_{h}\right\|_{0, \Omega}, \\
& \leq C \mu_{h} h^{\min \{s, k\}}\|(\mathbf{u}, 0)\|_{s} \nu^{\frac{1}{2}} h^{-1}\left\|\mathbf{v}_{h}\right\|_{0, \Omega} .
\end{aligned}
$$

Similarly,

$$
\begin{aligned}
\left|T_{2}\right| & \leq \sum_{K \in \mathcal{T}}\|\beta\|_{L^{\infty}(K)^{d}}\left\|\xi_{u}\right\|_{0, K}\left\|\nabla \mathbf{v}_{h}\right\|_{0, K} \\
& \leq C\|\beta\|_{L^{\infty}(\Omega)^{d}} h^{\min \{s, k\}}|u|_{s+1, \Omega}\left\|\mathbf{v}_{h}\right\|_{0, \Omega} \\
& \leq C \mu_{h} h^{\min \{s, k\}} \nu^{\frac{1}{2}}|u|_{s+1, \Omega} \nu^{\frac{1}{2}} h^{-1}\left\|\mathbf{v}_{h}\right\|_{0, \Omega} \\
& \leq C \mu_{h} h^{\min \{s, k\}}\|(\mathbf{u}, 0)\|_{s} \nu^{\frac{1}{2}} h^{-1}\left\|\mathbf{v}_{h}\right\|_{0, \Omega},
\end{aligned}
$$

and

$$
\begin{aligned}
\left|T_{3}\right| & \leq\left(\frac{\|\beta\|_{L^{\infty}(\Omega)^{d}} h^{\frac{1}{2}}}{\nu^{\frac{1}{2}}}\right)\left(\sum_{e \subset \mathcal{E}}\left\|\xi_{u}\right\|_{0, e}^{2}\right)^{\frac{1}{2}}\left|\mathbf{v}_{h}\right|_{c} \\
& \leq C \mu_{h} h^{\min \{s, k\}} \nu^{\frac{1}{2}}|\mathbf{u}|_{s+1, \Omega}\left|\mathbf{v}_{h}\right|_{c} \\
& \leq C \mu_{h} h^{\min \{s, k\}}\|(\mathbf{u}, 0)\|_{s}\left|\mathbf{v}_{h}\right|_{c} .
\end{aligned}
$$

Here, $\mathcal{E}=\mathcal{E}_{\mathcal{I}} \cup \mathcal{E}_{\mathcal{D}}$. This completes the proof of the first estimate. The second and third estimates can be proved in a similar way.

Finally, the last set of estimates can be proved easily by noting that, for $\mathbf{w} \in$ $H_{0}^{1}(\Omega)^{d}$, we have

$$
\begin{aligned}
b(\mathbf{w}, \underline{\sigma}) & =-\int_{\Omega} \nabla \mathbf{w}: \underline{\sigma} d \mathbf{x}, \\
\mathcal{O}(\mathbf{v}, \mathbf{w}) & =\int_{\Omega}((\gamma-\nabla \cdot \beta) \mathbf{v} \cdot \mathbf{w}-\mathbf{v} \cdot \nabla \mathbf{w} \cdot \beta) d \mathbf{x} .
\end{aligned}
$$

This completes the proof of Lemma 4.1 .

4.2. Step 2: A first estimate of the $\mathcal{A}$-seminorm. Now, let us keep $\underline{\Pi}, \boldsymbol{\Pi}$ and $\Pi$ as in the previous lemma, and let us split the error $\left(\underline{\sigma}-\underline{\sigma}_{h}, \mathbf{u}-\mathbf{u}_{h}, p-p_{h}\right)$ as follows:

$$
\left(\underline{\sigma}-\underline{\sigma}_{h}, \mathbf{u}-\mathbf{u}_{h}, p-p_{h}\right)=\left(\underline{\xi}_{\sigma}, \xi_{u}, \xi_{p}\right)+\left(\underline{\eta}_{\sigma}, \eta_{u}, \eta_{p}\right),
$$

where again $\left(\underline{\xi}_{\sigma}, \xi_{u}, \xi_{p}\right)=(\underline{\sigma}-\underline{\Pi \sigma}, \mathbf{u}-\Pi \mathbf{\Pi}, p-\Pi p)$ and $\left(\underline{\eta}_{\sigma}, \eta_{u}, \eta_{p}\right)=\left(\underline{\Pi \sigma}-\underline{\sigma}_{h}, \mathbf{\Pi u}-\right.$ $\left.\mathbf{u}_{h}, \Pi p-p_{h}\right)$.

We have the following result.

Lemma 4.2. We have

$$
\left|\left(\underline{\eta}_{\sigma}, \eta_{u}, \eta_{p}\right)\right|_{\mathcal{A}}^{2} \leq \Psi_{1} h^{\min \{s, k\}}\|(\mathbf{u}, p)\|_{s},
$$

where

$$
\Psi_{1}=C_{1}\left|\left(\underline{\eta}_{\sigma}, \eta_{u}, \eta_{p}\right)\right|_{\mathcal{A}}+C_{\mathcal{O}} \mu_{h} \nu^{\frac{1}{2}} h^{-1}\left\|\eta_{u}\right\|_{0, \Omega},
$$

and $C_{1}=2 C_{b}+C_{c}+2 C_{d}+C_{e}+C_{\mathcal{O}} \mu_{h}$. 
Let us emphasize that this result holds even if the function $\gamma_{0}$, defined by (1.2), is identically equal to zero. If we assume that $\gamma_{0} \geq \gamma_{\star}>0$, the $\mathcal{A}$-seminorm can be bounded easily in terms of $h^{\min \{s, k\}}\|(\mathbf{u}, p)\|_{s}$ only.

Proof. By the definition of the $\mathcal{A}$-seminorm, (2.14), and Galerkin orthogonality, we have

$$
\left|\left(\underline{\eta}_{\sigma}, \eta_{u}, \eta_{p}\right)\right|_{\mathcal{A}}^{2}=\mathcal{A}\left(\underline{\xi}_{\sigma}, \xi_{u}, \xi_{p} ; \underline{\eta}_{\sigma}, \eta_{u}, \eta_{p}\right) .
$$

Now, a direct application of Lemma 4.1 gives

$$
\left|\left(\underline{\eta}_{\sigma}, \eta_{u}, \eta_{p}\right)\right|_{\mathcal{A}}^{2} \leq \Theta h^{\min \{s, k\}}\|(\mathbf{u}, p)\|_{s},
$$

where

$$
\begin{aligned}
\Theta= & C_{b}\left\|\underline{\eta}_{\sigma}\right\|_{a}+\left(C_{b}+C_{c}+C_{d}+C_{\mathcal{O}} \mu_{h}\right)\left|\eta_{u}\right|_{c} \\
& +\left(C_{e}+C_{d}\right)\left|\eta_{p}\right|_{e}+C_{\mathcal{O}} \mu_{h} \nu^{\frac{1}{2}} h^{-1}\left\|\eta_{u}\right\|_{0, \Omega} \leq \Psi_{1} .
\end{aligned}
$$

Note that we also used that $a\left(\underline{\xi}_{\sigma}, \underline{\eta}_{\sigma}\right)=0$ thanks to the orthogonality properties of the $L^{2}$-projections. This completes the proof.

It is clear that when estimating the error in the $\mathcal{A}$-seminorm, we must find an estimate of the $L^{2}$-norm of the error in the velocity. This is achieved by a standard duality argument as we show next.

4.3. Step 3: A key estimate of negative-order norms. The so-called dual problem is the following:

$$
\begin{aligned}
& -\nu \Delta \mathbf{z}-\nabla \cdot(\mathbf{z} \otimes \beta)+\gamma \mathbf{z}-\nabla \psi=\lambda \quad \text { in } \Omega, \\
& -\nabla \cdot \mathbf{z}=\chi \quad \text { in } \Omega, \\
& \mathbf{z}=\mathbf{0} \quad \text { on } \Gamma \text {, }
\end{aligned}
$$

where the data $\chi$ satisfies the usual compatibility condition $\int_{\Omega} \chi d \mathbf{x}=0$. Due to assumption (1.2) on $\gamma_{0}$, it can be easily seen that the above problem has a unique solution in $H_{0}^{1}(\Omega)^{d} \times L_{0}^{2}(\Omega)$.

Now let $\Gamma$ be of class $C^{\ell+2}$ for $\ell \geq 0$. From the regularity results in [4, 18, 27, we conclude that for $\lambda \in H^{\ell}(\Omega)^{d}, \chi \in H^{\ell+1}(\Omega)$ we have $\mathbf{z} \in H^{\ell+2}(\Omega)^{d}, \psi \in H^{\ell+1}(\Omega)$ and the stability estimate

$$
\|(\mathbf{z}, \psi)\|_{\ell+1} \leq C_{\text {stab }}\|(\chi, \lambda)\|_{\ell},
$$

where we use the analogous notation

$$
\|(\chi, \lambda)\|_{\ell}=\nu^{\frac{1}{2}}\|\chi\|_{\ell+1, \Omega}+\nu^{-\frac{1}{2}}\|\lambda\|_{\ell, \Omega},
$$

and where the constant $C_{\text {stab }}$ depends on dimensionless quantities as can be seen from a simple scaling argument.

Lemma 4.3. We have, for $\ell \geq 0$,

$$
\int_{\Omega} \lambda \cdot \mathbf{e}_{u} d \mathbf{x}+\int_{\Omega} \chi e_{p} d \mathbf{x} \leq C_{\text {stab }} \Psi_{2} h^{\min \{\ell+1, k\}}\|(\chi, \lambda)\|_{\ell},
$$

where

$$
\Psi_{2}=C_{1}\left|\left(\underline{\eta}_{\sigma}, \eta_{u}, \eta_{p}\right)\right|_{\mathcal{A}}+C_{\mathcal{O}} \mu_{h} \nu^{\frac{1}{2}} h^{-1}\left\|\eta_{u}\right\|_{0, \Omega}+C_{2} h^{\min \{s, k\}}\|(\mathbf{u}, p)\|_{s},
$$

and $C_{2}=C_{a}^{2}+2 C_{b}^{2}+C_{c}^{2}+2 C_{d}^{2}+C_{e}^{2}+C_{\mathcal{O}}^{2} \mu_{h}$. 
Proof. If we introduce the auxiliary variable $\underline{\zeta}=-\nu \nabla \mathbf{z}$, it is easy to verify that we have

$$
\begin{aligned}
a(\underline{\zeta}, \underline{\tau})-b(\mathbf{z}, \underline{\tau}) & =0 & & \forall \underline{\tau} \in \underline{\Sigma}, \\
b(\mathbf{v}, \underline{\zeta})+c(\mathbf{z}, \mathbf{v})-d(\mathbf{v}, \psi)+\mathcal{O}(\mathbf{v}, \mathbf{z}) & =\int_{\Omega} \lambda \cdot \mathbf{v} d \mathbf{x} & & \forall \mathbf{v} \in \mathbf{V}, \\
d(\mathbf{z}, q)+e(\psi, q) & =\int_{\Omega} \chi q d \mathbf{x} & & \forall q \in Q .
\end{aligned}
$$

Now, if we add the above equations, we obtain

$$
\int_{\Omega} \lambda \cdot \mathbf{v} d \mathbf{x}+\int_{\Omega} \chi q d \mathbf{x}=\mathcal{A}(\underline{\tau}, \mathbf{v}, q ; \underline{\zeta}, \mathbf{z}, \psi) \quad \forall(\underline{\tau}, \mathbf{v}, q) \in \underline{\Sigma} \times \mathbf{V} \times Q
$$

and if we take $(\underline{\tau}, \mathbf{v}, q)=\left(\underline{e}_{\sigma}, \mathbf{e}_{u}, e_{p}\right)$, and use the Galerkin orthogonality property, we get

$$
\int_{\Omega} \lambda \cdot \mathbf{e}_{u} d \mathbf{x}+\int_{\Omega} \chi e_{p} d \mathbf{x}=\mathcal{A}\left(\underline{e}_{\sigma}, \mathbf{e}_{u}, e_{p} ; \underline{\zeta}-\underline{\zeta}_{h}, \mathbf{z}-\mathbf{z}_{h}, \psi-\psi_{h}\right)
$$

for any $\left(\underline{\zeta}_{h}, \mathbf{z}_{h}, \psi_{h}\right) \in \underline{\Sigma}_{h} \times \mathbf{V}_{h} \times Q_{h}$. Finally, if we take $\underline{\zeta}_{h}=\underline{\Pi} \underline{\zeta}, \mathbf{z}_{h}=\Pi \mathbf{z}$, and $\psi_{h}=\Pi \psi$, we obtain

$$
\begin{aligned}
\int_{\Omega} \lambda \cdot \mathbf{e}_{u} d \mathbf{x}+\int_{\Omega} \chi e_{p} d \mathbf{x} & =\mathcal{A}\left(\underline{e}_{\sigma}, \mathbf{e}_{u}, e_{p} ; \underline{\xi}_{\zeta}, \xi_{z}, \xi_{\psi}\right) \\
& =\mathcal{A}\left(\underline{\eta}_{\sigma}, \eta_{u}, \eta_{p} ; \underline{\xi}_{\zeta}, \xi_{z}, \xi_{\psi}\right)+\mathcal{A}\left(\underline{\xi}_{\sigma}, \xi_{u}, \xi_{p} ; \underline{\xi}_{\zeta}, \xi_{z}, \xi_{\psi}\right) .
\end{aligned}
$$

As in Lemma 4.2, we have

$$
\left|\mathcal{A}\left(\underline{\eta}_{\sigma}, \eta_{u}, \eta_{p} ; \underline{\xi}_{\zeta}, \xi_{z}, \xi_{\psi}\right)\right| \leq \Theta_{1} h^{\min \{\ell+1, k\}}\|(\mathbf{z}, \psi)\|_{\ell+1},
$$

where $\Theta_{1}=C_{1}\left|\left(\underline{\eta}_{\sigma}, \eta_{u}, \eta_{p}\right)\right|_{\mathcal{A}}+C_{\mathcal{O}} \mu_{h} \nu^{\frac{1}{2}} h^{-1}\left\|\eta_{u}\right\|_{0, \Omega}$, and, by a direct application of Lemma 4.1,

$$
\left|\mathcal{A}\left(\underline{\xi}_{\sigma}, \xi_{u}, \xi_{p} ; \underline{\xi}_{\zeta}, \xi_{z}, \xi_{\psi}\right)\right| \leq \Theta_{2} h^{\min \{\ell+1, k\}}\|(\mathbf{z}, \psi)\|_{\ell+1},
$$

where $\Theta_{2}=C_{2} h^{\min \{s, k\}}\|(u, p)\|_{s}$. As a consequence, by the stability estimate (4.3),

$$
\int_{\Omega} \lambda \cdot \mathbf{e}_{u} d \mathbf{x}+\int_{\Omega} \chi e_{p} d \mathbf{x} \leq C_{\text {stab }}\left(\Theta_{1}+\Theta_{2}\right) h^{\min \{\ell+1, k\}}\|(\chi, \lambda)\|_{\ell} .
$$

Since $\Psi_{2}=\Theta_{1}+\Theta_{2}$, this completes the proof.

4.4. Step 4: The estimates of the $\mathcal{A}$-seminorm and negative-order norms. An immediate consequence of Lemma 4.3 is the following result.

Corollary 4.4. Assume that $C_{\mathrm{stab}} C_{\mathcal{O}} \mu_{h}<1$. Then we have

$$
\nu^{\frac{1}{2}} h^{-1}\left\|\eta_{u}\right\|_{0, \Omega} \leq \frac{C_{\text {stab }}}{1-C_{\text {stab }} C_{\mathcal{O}} \mu_{h}}\left(C_{1}\left|\left(\underline{\eta}_{\sigma}, \eta_{u}, \eta_{p}\right)\right|_{\mathcal{A}}+C_{2} h^{\min \{s, k\}}\|(\mathbf{u}, p)\|_{s}\right) .
$$

Proof. This result follows easily from Lemma 4.3 with $\ell=0$ simply by taking $(\chi, \lambda)=\left(0, \nu^{\frac{1}{2}} \eta_{u}\right)$ and noting that, in this case, $\|(\chi, \lambda)\|_{0}=\left\|\eta_{u}\right\|_{0, \Omega}$ and also $\int_{\Omega} \lambda \cdot \mathbf{e}_{u} d \mathbf{x}=\nu^{\frac{1}{2}}\left\|\eta_{u}\right\|_{0, \Omega}^{2}$.

We can now obtain the error estimates in the $\mathcal{A}$-seminorm and in negative-order norms given by Theorem 3.1 . 
Corollary 4.5. Assume that $C_{\text {stab }} C_{\mathcal{O}} \mu_{h}<1$. Then we have

$$
\begin{aligned}
& \left|\left(\underline{\eta}_{\sigma}, \eta_{u}, \eta_{p}\right)\right|_{\mathcal{A}} \leq C_{3} h^{\min \{s, k\}}\|(\mathbf{u}, p)\|_{s}, \\
& \left\|\left(\mathbf{e}_{u}, e_{p}\right)\right\|_{-\ell-1} \leq C_{4} h^{\min \{\ell+1, k\}+\min \{s, k\}}\|(\mathbf{u}, p)\|_{s}, \quad \forall \ell \geq 0,
\end{aligned}
$$

where

$$
\begin{aligned}
& C_{3}=\left(C_{1}+\sqrt{2 C_{2}}\right) /\left(1-C_{\text {stab }} C_{\mathcal{O}} \mu_{h}\right), \\
& C_{4}=2 C_{\text {stab }}\left(C_{1} C_{3}+C_{2}\right) /\left(1-C_{\text {stab }} C_{\mathcal{O}} \mu_{h}\right) .
\end{aligned}
$$

Note that from this result, the first and third estimates of Theorem 3.1 easily follow.

Proof. If we insert the estimate of the $L^{2}$-norm of the projection of the error in the velocity obtained in Corollary 4.4 into the estimate of Lemma 4.2, we get

$$
\left|\left(\underline{\eta}_{\sigma}, \eta_{u}, \eta_{p}\right)\right|_{\mathcal{A}}^{2} \leq \Psi h^{\min \{s, k\}}\|(\mathbf{u}, p)\|_{s},
$$

where

$$
\Psi=\frac{1}{1-C_{\text {stab }} C_{\mathcal{O}} \mu_{h}}\left(C_{1}\left|\left(\underline{\eta}_{\sigma}, \eta_{u}, \eta_{p}\right)\right|_{\mathcal{A}}+C_{2} h^{\min \{s, k\}}\|(\mathbf{u}, p)\|_{s}\right) .
$$

The first estimate now follows after a simple application of Young's inequality.

If we now insert the first estimate in the inequality given by Corollary 4.4, we get

$$
\nu^{\frac{1}{2}} h^{-1}\left\|\eta_{u}\right\|_{0, \Omega} \leq \frac{C_{4}}{2} h^{\min \{s, k\}}\|(\mathbf{u}, p)\|_{s} .
$$

Finally, the second estimate follows by inserting the first estimate of the corollary and the above inequality in the estimate given by Lemma 4.3 and by noting that

$$
\nu^{\frac{1}{2}}\left\|\mathbf{e}_{u}\right\|_{-\ell, \Omega}=\sup _{\lambda \in\left(\mathcal{C}_{0}^{\infty}(\Omega)\right)^{d}} \frac{\int_{\Omega} \mathbf{e}_{u} \cdot \lambda d x}{\nu^{-\frac{1}{2}}\|\lambda\|_{\ell, \Omega}}
$$

and that

$$
\nu^{-\frac{1}{2}}\left\|e_{p}\right\|_{-\ell-1, \Omega}=\sup _{\chi \in \mathcal{C}_{0}^{\infty}(\Omega) \cap L_{0}^{2}(\Omega)} \frac{\int_{\Omega} e_{p} \chi d x}{\nu^{\frac{1}{2}}\|\chi\|_{\ell+1, \Omega}} .
$$

This completes the proof of the corollary.

4.5. Step 5: Estimate of the $L^{2}$-norm of the error in the pressure. To prove Theorem 3.1, it only remains to get the estimate of the error in the pressure. To do that, we use a variation of the corresponding argument presented in [12].

Since the error in the pressure, $e_{p}$, belongs to $L^{2}(\Omega)$ and is such that $\int_{\Omega} e_{p} d \mathbf{x}=0$, it follows from the continuous inf-sup condition in (3.11) that there is a velocity field $\mathbf{w} \in H_{0}^{1}(\Omega)^{d}$ satisfying

$$
-\int_{\Omega} e_{p} \nabla \cdot \mathbf{w} d \mathbf{x} \geq \kappa\left\|e_{p}\right\|_{0, \Omega}^{2}, \quad\|\mathbf{w}\|_{1, \Omega} \leq\left\|e_{p}\right\|_{0, \Omega} .
$$

In the following result, we exploit this fact to obtain an upper bound of the $L^{2}$-norm of $e_{p}$ in terms of the bilinear forms of the LDG method.

Lemma 4.6. Let $\mathbf{w} \in H_{0}^{1}(\Omega)^{d}$ be the continuous velocity field in (4.4). Then we have

$$
\kappa\left\|e_{p}\right\|_{0, \Omega}^{2} \leq \mathcal{A}\left(\underline{e}_{\sigma}, \mathbf{e}_{u}, e_{p} ; \underline{0}, \xi_{w}, 0\right)+b\left(\mathbf{w}, \underline{e}_{\sigma}\right)-\mathcal{O}\left(\mathbf{e}_{u}, \mathbf{w}\right)
$$


Proof. By the first inequality in (4.4), we have

$$
\begin{aligned}
\kappa\left\|e_{p}\right\|_{0, \Omega}^{2} & \leq-\int_{\Omega} e_{p} \nabla \cdot \mathbf{w} d \mathbf{x}=d\left(\mathbf{w}, e_{p}\right) \\
& =\left[-b\left(\mathbf{w}, \underline{e}_{\sigma}\right)+c\left(\mathbf{e}_{u}, \mathbf{w}\right)+d\left(\mathbf{w}, e_{p}\right)+\mathcal{O}\left(\mathbf{e}_{u}, \mathbf{w}\right)\right]+b\left(\mathbf{w}, \underline{e}_{\sigma}\right)-\mathcal{O}\left(\mathbf{e}_{u}, \mathbf{w}\right) \\
& =\mathcal{A}\left(\underline{e}_{\sigma}, \mathbf{e}_{u}, e_{p} ; \underline{0}, \mathbf{w}, 0\right)+b\left(\mathbf{w}, \underline{e}_{\sigma}\right)-\mathcal{O}\left(\mathbf{e}_{u}, \mathbf{w}\right) \\
& =\mathcal{A}\left(\underline{e}_{\sigma}, \mathbf{e}_{u}, e_{p} ; \underline{0}, \xi_{w}, 0\right)+b\left(\mathbf{w}, \underline{e}_{\sigma}\right)-\mathcal{O}\left(\mathbf{e}_{u}, \mathbf{w}\right),
\end{aligned}
$$

by Galerkin orthogonality. Note that we have used the fact that $c\left(\mathbf{e}_{u}, \mathbf{w}\right)=0$ since $\mathbf{w} \in H_{0}^{1}(\Omega)^{d}$. This completes the proof.

Next, we use the upper bounds of Lemma 4.1 and the already obtained bounds on the errors to obtain the wanted estimate.

Lemma 4.7. We have

$$
\nu^{-\frac{1}{2}}\left\|e_{p}\right\|_{0, \Omega} \leq \frac{C_{5}}{\kappa} h^{\min \{s, k\}}\|(\mathbf{u}, p)\|_{s},
$$

where

$$
C_{5}=C_{\mathcal{A}}+\left(M_{h}+\mu_{h}\right) C_{4}+\left(C_{1} C_{3}+C_{2}\right)\left(\frac{1+C_{\mathrm{stab}} C_{\mathcal{O}} \mu_{h}}{1-C_{\mathrm{stab}} C_{\mathcal{O}} \mu_{h}}\right) .
$$

Proof. To prove this result, we proceed as follows:

$$
\begin{aligned}
\kappa\left\|e_{p}\right\|_{0, \Omega}^{2} \leq & \mathcal{A}\left(\underline{\eta}_{\sigma}, \eta_{u}, \eta_{p} ; \underline{0}, \xi_{w}, 0\right)+\mathcal{A}\left(\underline{\xi}_{\sigma}, \xi_{u}, \xi_{p} ; \underline{0}, \xi_{w}, 0\right) & & \\
& +b\left(\mathbf{w}, \underline{e}_{\sigma}\right)-\mathcal{O}\left(\mathbf{e}_{u}, \mathbf{w}\right), & & \text { by the previous lemma, } \\
\leq & C_{5} h^{\min \{s, k\}}\|(\mathbf{u}, p)\|_{s} \nu^{\frac{1}{2}}\|\mathbf{w}\|_{1, \Omega} & & \text { by Lemma 4.1 } \\
\leq & C_{5} h^{\min \{s, k\}}\|(\mathbf{u}, p)\|_{s} \nu^{\frac{1}{2}}\left\|e_{p}\right\|_{0, \Omega}, & &
\end{aligned}
$$

by Lemma 4.6 Lemma 4.1, and the second inequality in (4.4). This completes the proof.

Since the corollary we just proved is exactly the $L^{2}$-estimate of the pressure, the proof of Theorem 3.1 is complete.

\section{Numerical Results}

The numerical experiments in this section are devised to verify our theoretical estimates. As exact solution in our tests, we choose the two-dimensional analytical solution of the incompressible Navier-Stokes equations derived by Kovasznay in [19]. Since this solution is smooth for any Reynolds number Re, it allows us to easily assess the approximation properties of the LDG method for higher-order elements and for a wide range of the Reynolds number.

Let us describe Kovasznay's solution for a given Reynolds number Re: let the (dimensionless) viscosity $\nu=1 / R e$, and set $\lambda=R e / 2-\sqrt{R e^{2} / 4+4 \pi^{2}}$. Then, the functions

$$
\begin{aligned}
u_{1}(x, y) & =1-e^{\lambda x} \cos 2 \pi y, \\
u_{2}(x, y) & =\frac{\lambda}{2 \pi} e^{\lambda x} \sin 2 \pi y, \\
p(x, y) & =\frac{1}{2} e^{2 \lambda x}+C,
\end{aligned}
$$


TABLE $1 . L^{2}$-errors and numerica $l$ convergence rates for $R e=10$.

\begin{tabular}{|c|c|cc|cc|cc|}
\hline$k$ & level & $\left\|\mathbf{e}_{u}\right\|_{0, \Omega}$ & order & $\left\|e_{p}\right\|_{0, \Omega}$ & order & $\left\|\underline{e}_{\sigma}\right\|_{0, \Omega}$ & order \\
\hline \multirow{3}{*}{1} & 5 & $1.5 \mathrm{e}-02$ & 2.10 & $5.9 \mathrm{e}-02$ & 1.23 & $1.3 \mathrm{e}-01$ & 0.84 \\
& 6 & $3.7 \mathrm{e}-03$ & 2.06 & $2.9 \mathrm{e}-02$ & 1.03 & $7.1 \mathrm{e}-02$ & 0.91 \\
& 7 & $9.2 \mathrm{e}-04$ & 2.01 & $1.5 \mathrm{e}-02$ & 0.99 & $3.7 \mathrm{e}-02$ & 0.96 \\
\hline \multirow{3}{*}{2} & 5 & $4.2 \mathrm{e}-04$ & 3.11 & $1.2 \mathrm{e}-03$ & 2.73 & $1.6 \mathrm{e}-03$ & 2.50 \\
& 6 & $5.1 \mathrm{e}-05$ & 3.04 & $2.1 \mathrm{e}-04$ & 2.49 & $3.3 \mathrm{e}-04$ & 2.28 \\
& 7 & $6.3 \mathrm{e}-06$ & 3.01 & $4.6 \mathrm{e}-05$ & 2.23 & $7.5 \mathrm{e}-05$ & 2.13 \\
\hline \multirow{3}{*}{3} & 5 & $1.7 \mathrm{e}-05$ & 3.92 & $9.6 \mathrm{e}-05$ & 2.87 & $2.0 \mathrm{e}-04$ & 2.69 \\
& 6 & $1.0 \mathrm{e}-06$ & 4.04 & $1.3 \mathrm{e}-05$ & 2.87 & $2.9 \mathrm{e}-05$ & 2.81 \\
& 7 & $6.1 \mathrm{e}-08$ & 4.05 & $1.7 \mathrm{e}-06$ & 2.95 & $3.9 \mathrm{e}-06$ & 2.92 \\
\hline \multirow{3}{*}{4} & 4 & $2.6 \mathrm{e}-06$ & 5.87 & $1.2 \mathrm{e}-05$ & 4.88 & $2.0 \mathrm{e}-05$ & 4.94 \\
& 5 & $4.6 \mathrm{e}-08$ & 5.83 & $4.6 \mathrm{e}-07$ & 4.74 & $8.2 \mathrm{e}-07$ & 4.60 \\
& 6 & $9.5 \mathrm{e}-10$ & 5.59 & $2.2 \mathrm{e}-08$ & 4.40 & $4.1 \mathrm{e}-08$ & 4.31 \\
\hline
\end{tabular}

solve the Oseen equations (1.1) with $\beta=\mathbf{u}, \gamma=0$, a suitably chosen right-hand side $\mathbf{f}$ and a constant $C$ chosen such that $\int_{\Omega} p d \mathbf{x}=0$. In our numerical experiments, we take the computational domain to be

$$
\Omega=\left(-\frac{1}{2}, \frac{3}{2}\right) \times(0,2)
$$

and impose Dirichlet boundary values for the velocities on $\Gamma$ obtained from (5.1). The vector plot on the left-hand side of Figure 1 shows that this flow is not trivial. We emphasize that with our choice of $\beta$ and $\gamma$ we have that $\gamma_{0} \equiv 0$ in (1.2); thus, we test the sharpness of the theoretical results as well as the performance of the LDG method in this difficult limiting case.

The asymptotic behavior of the $L^{2}$-norms of the components $(\underline{\sigma}, \mathbf{u}, p)$ of the exact solution with respect to the Reynolds number is displayed on the right-hand side of Figure 1. When the Reynolds number becomes large, the $L^{2}$-norm of the pressure decays with $\mathcal{O}(1 / R e)$, while the $L^{2}$-norm of the velocity remains essentially constant if the Reynolds number is larger than about 30. The $L^{2}$-norm of the "stress" tensor $\underline{\sigma}=\nu \nabla \mathbf{u}$ also decays like $\mathcal{O}(1 / R e)$.

Our computations were performed on quadrilateral meshes generated by consecutive refinement of the original square. In each refinement step, each grid cell is divided into four similar cells by connecting the edge midpoints. Therefore, grid level $L$ corresponds to a mesh width $h_{L}=2^{1-L}$. All the unknowns are approximated with tensor product polynomials of degree $k \geq 1$ and, throughout, we choose the parameters of the LDG method as $c_{11}=\nu$ and $d_{11}=1 / 10 \nu$.

First, we display in Table 1 the $L^{2}$-norms of the errors $\mathbf{e}_{u}=\mathbf{u}-\mathbf{u}_{h}, e_{p}=p-p_{h}$ and $\underline{e}_{\sigma}=\underline{\sigma}-\underline{\sigma}_{h}$, respectively, for small Reynolds numbers, obtained on different grid levels and for different polynomial degrees. We see that the optimal approximation order $k+1$ is achieved for the velocities whereas the pressure $p$ and "stresses" $\underline{\sigma}$ converge with one order less, hereby confirming the sharpness of the error estimates in Theorem 3.1 For biquadratic elements, we display in Figure 2 the dependence of the $L^{2}$-norms of the errors $\mathbf{e}_{u}, \underline{e}_{\sigma}$, and $e_{p}$ on the Reynolds number. There, the norms are scaled with the appropriate powers of $\nu$ taken from the estimates in Theorem 3.1 The diagrams show the predicted convergence orders two and 


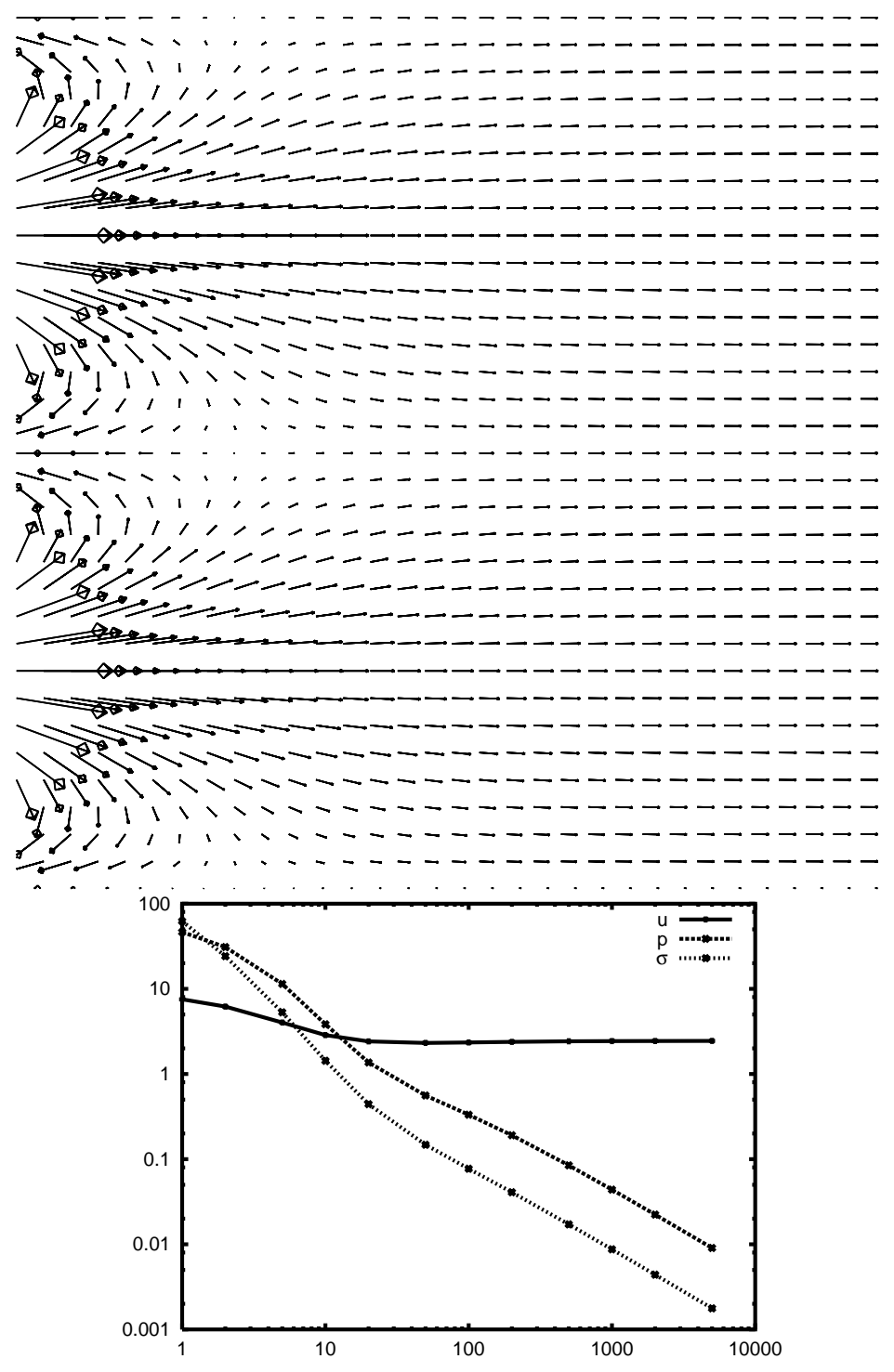

Figure 1 . Velocity field for $R e=10$ (top) and $L^{2}$-norms of solution components $(\underline{\sigma}, \mathbf{u}, p)$, depending on the Reynolds number (bottom).

three, respectively. Furthermore, robustness of the discretization with respect to the Reynolds number is evident.

Next, let us numerically verify the negative-order estimates obtained in Theorem 3.1. To this end, we consider the smooth linear function $M$ defined by

$$
M(u):=\int_{\Omega} u(\mathbf{x}) \eta(\mathbf{x}) d \mathbf{x}
$$



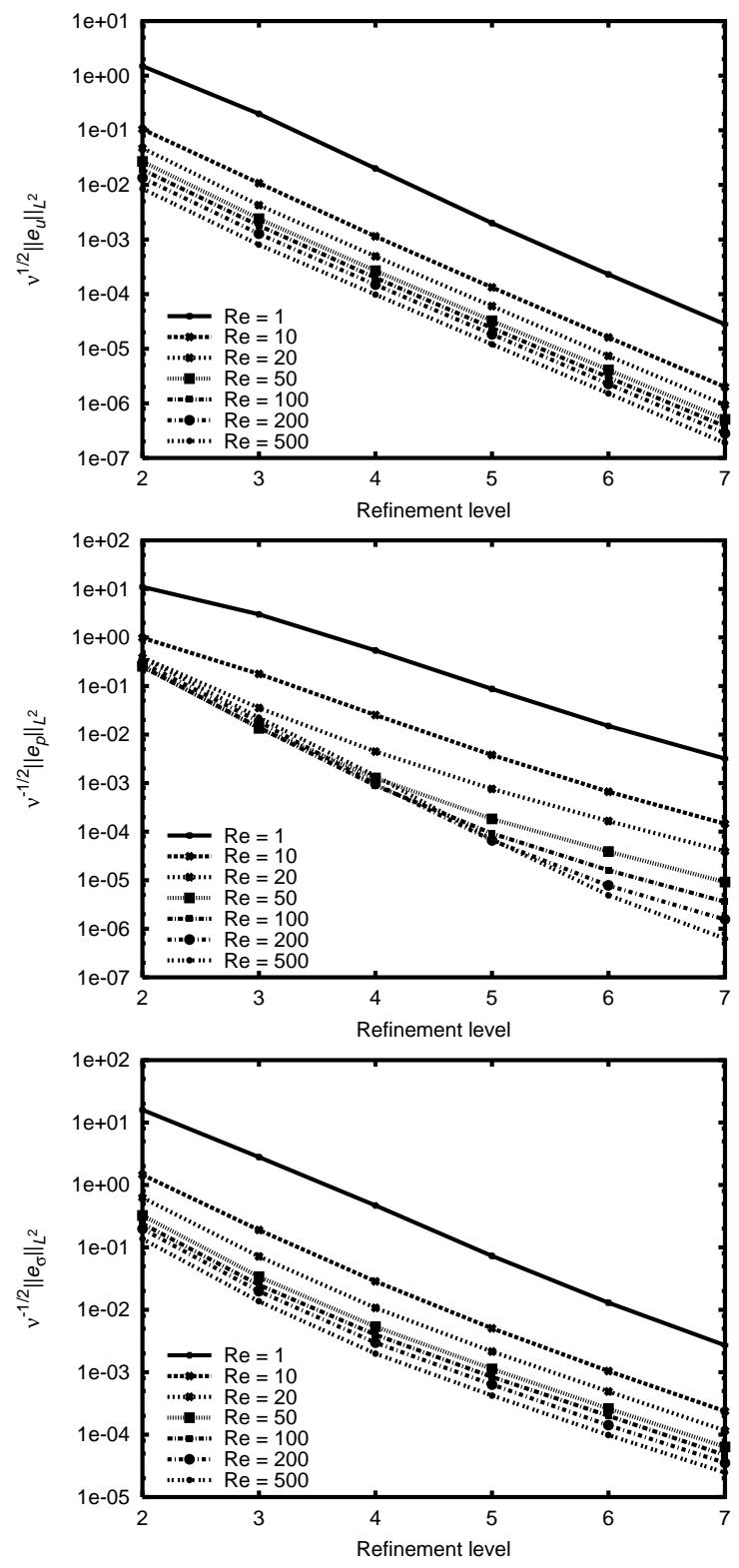

FiguRE 2. Scaled $L^{2}$-norms of the errors $\mathbf{e}_{u}, e_{p}$ and $\underline{e}_{\sigma}$ with $k=2$ for different Reynolds numbers.

where $\eta$ is the $C^{\infty}$-cutoff function on a ball of radius $\varrho=0.8$ defined by

$$
\eta(\mathbf{x}):=\left\{\begin{array}{ll}
\exp \left(-\frac{r^{2}}{r^{2}-\varrho^{2}}\right) & r<\varrho \\
0 & r \geq \varrho
\end{array} \quad \text { where } \quad r:=\left|\mathbf{x}-\left(\begin{array}{c}
0.5 \\
0
\end{array}\right)\right| .\right.
$$

We show in Figure 3 the errors of the weighted averages, $M\left(\mathbf{e}_{u}\right), M\left(e_{p}\right)$ and $M\left(\underline{e}_{\sigma}\right)$, for $k=2$, again scaled by the powers of $\nu$ indicated by our analytical 

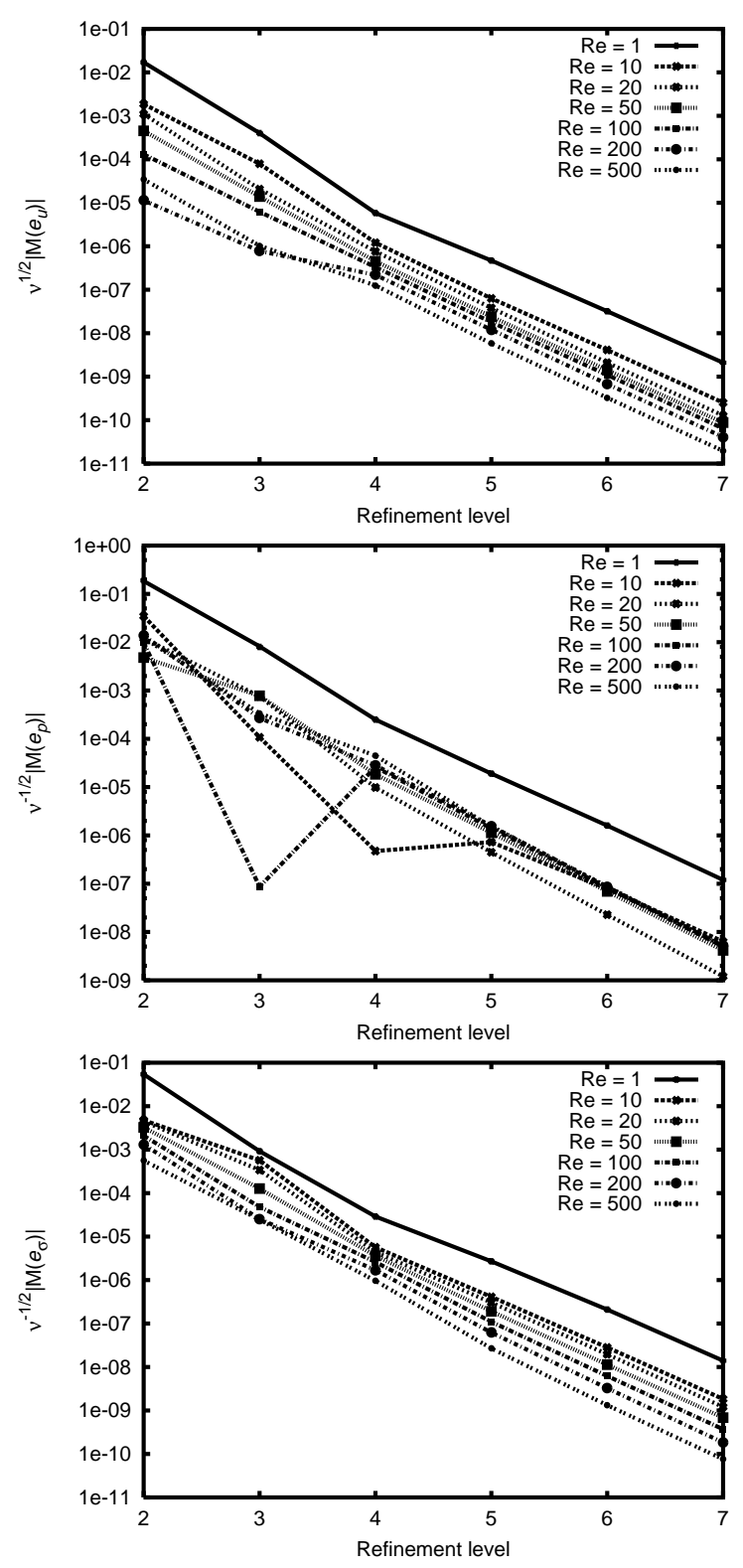

FiguRE 3. Weighted averages of $\mathbf{e}_{u}, e_{p}$ and $\underline{e}_{\sigma}$ with $k=2$ for different Reynolds numbers.

results. Since $\eta$ and the true solution $(\underline{\sigma}, \mathbf{u}, p)$ are smooth, we can exploit the negative-order estimate (3.16). We expect convergence of $M\left(\mathbf{e}_{u}\right)$ with at least fourth order. Since (3.16) establishes an estimate of the $H^{-2}$-norm of the pressure, we observe fourth-order convergence of $p$, too. In the last diagram, we even observe fourth-order convergence of the weighted average of $\underline{\sigma}$. We show in Table 2 the same evaluations for fixed Reynolds number $R e=20$ and for $k=2$. We do not show 
TABLE 2. Weighted averages of $\mathbf{e}_{u}, e_{p}$ and $\underline{e}_{\sigma}$ with Reynolds number $R e=20$ and biquadratic elements.

\begin{tabular}{|c|cc|cc|cc|}
\hline level & $\nu^{\frac{1}{2}}\left|M\left(\mathbf{e}_{u}\right)\right|$ & order & $\nu^{-\frac{1}{2}}\left|M\left(e_{p}\right)\right|$ & order & $\nu^{-\frac{1}{2}}\left|M\left(\underline{e}_{\sigma}\right)\right|$ & order \\
\hline 2 & $4.9 \mathrm{e}-03$ & 2.53 & $2.7 \mathrm{e}-03$ & 3.61 & $1.0 \mathrm{e}-03$ & 2.86 \\
3 & $9.2 \mathrm{e}-05$ & 5.72 & $1.7 \mathrm{e}-04$ & 3.95 & $7.6 \mathrm{e}-05$ & 3.72 \\
4 & $3.4 \mathrm{e}-06$ & 4.76 & $2.3 \mathrm{e}-06$ & 6.27 & $9.8 \mathrm{e}-07$ & 6.28 \\
5 & $1.7 \mathrm{e}-07$ & 4.34 & $1.0 \mathrm{e}-07$ & 4.43 & $6.8 \mathrm{e}-08$ & 3.85 \\
6 & $9.4 \mathrm{e}-09$ & 4.16 & $5.1 \mathrm{e}-09$ & 4.36 & $4.4 \mathrm{e}-09$ & 3.97 \\
7 & $5.7 \mathrm{e}-10$ & 4.06 & $2.7 \mathrm{e}-10$ & 4.22 & $2.7 \mathrm{e}-10$ & 4.00 \\
\hline
\end{tabular}

results for $k>2$ since the approximation is so good that the asymptotic regime is reached when the errors are in the range of machine accuracy.

\section{Concluding Remarks}

In this paper, we have extended the LDG method proposed for the Stokes system in [12] to the Oseen problem. We have shown, both theoretically as well as computationally, that optimal error estimates are obtained and that the resulting method performs well for a wide range of the Reynolds number.

From the analysis we have presented, it is not very difficult to realize that an LDG method that uses approximate velocities that are piecewise solenoidal can be easily defined. However, this choice has several interesting aspects that have to be properly discussed and analyzed. Such a case requires more space than allowed for this work and will be treated in a forthcoming paper.

\section{Appendix: The ImpaCt of the Choice $c_{11}=d_{11}^{-1}=\nu$ ON THE CONDITION NUMBERS OF THE LDG MATRICES}

The result we present here concerns the dependence of the condition numbers of the matrices for the velocity and the pressure with respect to the kinematic viscosity $\nu$ in the absence of convection.

In this case, it is easy to see that the weak formulation (2.12) induces the following matrix equation

$$
\left(\begin{array}{ccc}
A & B^{t} & 0 \\
-B^{t} & C & D^{t} \\
0 & -D & E
\end{array}\right)\left(\begin{array}{l}
\underline{S} \\
\mathbf{U} \\
P
\end{array}\right)=\left(\begin{array}{c}
\frac{F}{\mathbf{G}} \\
H
\end{array}\right)
$$

We have the following result.

Proposition A.1. If $c_{11}=d_{11}^{-1}=\nu$, the condition numbers of the matrices

$$
\mathbb{C}=C+B A^{-1} B^{t} \text { and } \mathbb{E}=E+D \mathbb{C}^{-1} D^{t},
$$

are independent of the kinematic viscosity $\nu$.

Proof. Since

$$
\mathbb{C}=C+B A^{-1} B^{t}=\nu\left(\frac{1}{\nu} C+B \frac{1}{\nu} A^{-1} B^{t}\right),
$$


and since the matrix $B$ is independent of $\nu$, the condition number of $\mathbb{C}$ is independent of $\nu$ if both $\frac{1}{\nu} C$ and $\nu A$ are independent of $\nu$. But, since we have

$$
\underline{S}^{t}(\nu A) \underline{T}=\int_{\Omega} \underline{\sigma}_{h}: \underline{\tau}_{h} d \mathbf{x},
$$

for the functions $\underline{\sigma}_{h}$ and $\underline{\tau}_{h}$ represented by $\underline{S}$ and $\underline{T}$, and

$$
\mathbf{U}^{t} \frac{1}{\nu} C \mathbf{V}=\int_{\mathcal{E}_{\mathcal{I}}} \frac{C_{11}}{\nu} \underline{\llbracket \mathbf{u}_{h} \rrbracket}: \llbracket \mathbf{v}_{h} \rrbracket d s+\int_{\mathcal{E}_{\mathcal{D}}} \frac{C_{11}}{\nu}\left(\mathbf{u}_{h} \otimes \mathbf{n}\right):\left(\mathbf{v}_{h} \otimes \mathbf{n}\right) d s,
$$

for the functions $\mathbf{u}_{h}$ and $\mathbf{v}_{h}$ represented by $\mathbf{U}$ and $\mathbf{V}$, it is clear that $\mathbb{C}$ is independent of $\nu$ if $\frac{C_{11}}{\nu}$ is independent of $\nu$. Thus, if $c_{11}=\nu$, we can write

$$
\mathbb{C}=\nu \tilde{\mathbb{C}},
$$

where $\tilde{\mathbb{C}}$ is independent of $\nu$; this implies that for this choice of $c_{11}$, the condition number of $\mathbb{C}$ is independent of $\nu$.

Now, since

$$
\mathbb{E}=E+D \mathbb{C}^{-1} D^{t}=\frac{1}{\nu}\left(\nu E+D \tilde{\mathbb{C}}^{-1} D^{t}\right),
$$

and since the matrices $D$ and $\tilde{\mathbb{C}}$ are independent of $\nu$, the condition number of $\mathbb{E}$ is also independent of $\nu$ if $\nu E$ is independent of $\nu$. But, since we have for $P$ corresponding to the function $p_{h}$

$$
P^{t}(\nu E) P=\nu e\left(p_{h}, p_{h}\right)=\int_{\mathcal{E}_{\mathcal{I}}} \nu D_{11}\left|\llbracket p_{h} \rrbracket\right|^{2} d s,
$$

this happens if $\nu D_{11}$ is independent of $\nu$. Thus, for $d_{11}=\nu^{-1}$ the condition number of $\mathbb{E}$ is independent of $\nu$. This completes the proof.

\section{REFERENCES}

1. D. N. Arnold, F. Brezzi, B. Cockburn, and L. D. Marini, Unified analysis of discontinuous Galerkin methods for elliptic problems, SIAM J. Numer. Anal. 39 (2001), 1749-1779. MR 2002k:65183

2. I. Babuška, C.E. Baumann, and J.T. Oden, A discontinuous hp-finite element method for diffusion problems: 1-D analysis, Comput. Math. Appl. 37 (1999), 103-122. MR 2000a:65118

3. G.A. Baker, Finite element methods for elliptic equations using nonconforming elements, Math. Comp. 31 (1977), 45-59. MR 55:4737

4. G.A. Baker, W.N. Jureidini, and O.A. Karakashian, Piecewise solenoidal vector fields and the Stokes problem, SIAM J. Numer. Anal. 27 (1990), 1466-1485. MR 91m:65246

5. C.E. Baumann and J.T. Oden, A discontinuous hp-finite element method for convectiondiffusion problems, Comput. Methods Appl. Mech. Engrg. 175 (1999), 311-341. MR 2000d:65171

6. C.E. Baumann and T.J. Oden, A discontinuous hp-finite element method for the solution of the Euler and Navier-Stokes equations, Internat. J. Numer. Methods in Fluids 31 (1999), 79-95. MR 2000g:76072

7. F. Brezzi and M. Fortin, Mixed and hybrid finite element methods, Springer Series in Computational Mathematics, vol. 15, Springer, New York, 1991. MR 92d:65187

8. P. Castillo, Performance of discontinuous Galerkin methods for elliptic partial differential equations, SIAM J. Sci. Comput., to appear.

9. P. Castillo, B. Cockburn, I. Perugia, and D. Schötzau, An a priori error analysis of the local discontinuous Galerkin method for elliptic problems, SIAM J. Numer. Anal. 38 (2000), 1676-1706. MR 2002k:65175

10. P.G. Ciarlet, The finite element method for elliptic problems, North-Holland, Amsterdam, 1978. MR 58:25001 
11. B. Cockburn, G. Kanschat, I. Perugia, and D. Schötzau, Superconvergence of the local discontinuous Galerkin method for elliptic problems on Cartesian grids, SIAM J. Numer. Anal. 39 (2001), 264-285. MR 2002g:65140

12. B. Cockburn, G. Kanschat, D. Schötzau, and C. Schwab, Local discontinuous Galerkin methods for the Stokes system, SIAM J. Numer. Anal. 40 (2002), 319-343.

13. B. Cockburn and C.-W. Shu, The local discontinuous Galerkin method for time-dependent convection-diffusion systems, SIAM J. Numer. Anal. 35 (1998), 2440-2463. MR 99j:65163

14. _ Runge-Kutta discontinuous Galerkin methods for convection-dominated problems, J. Sci. Comput. 16 (2001), 173-261. MR 2002i:65099

15. V. Girault and P.A. Raviart, Finite element methods for Navier-Stokes equations, Springer, New York, 1986. MR 88b:65129

16. P. Hansbo and M.G. Larson, Discontinuous finite element methods for incompressible and nearly incompressible elasticity by use of Nitsche's method, Comput. Methods Appl. Mech. Engrg. 191 (2002), 1895-1908.

17. P. Houston, C. Schwab, and E. Süli, Discontinuous $h p$-finite element methods for advectiondiffusion-reaction problems, SIAM J. Numer. Anal. 39 (2002), 2133-2163.

18. O.A. Karakashian and W.N. Jureidini, A nonconforming finite element method for the stationary Navier-Stokes equations, SIAM J. Numer. Anal. 35 (1998), 93-120. MR 99d:65320

19. L. I. G. Kovasznay, Laminar flow behind a two-dimensional grid, Proc. Camb. Philos. Soc. 44 (1948), 58-62. MR 9:476d

20. P. LeSaint and P.A. Raviart, On a finite element method for solving the neutron transport equation, Mathematical Aspects of Finite Elements in Partial Differential Equations (C. de Boor, ed.), Academic Press, New York, 1974, pp. 89-145. MR 58:31918

21. J.-G. Liu and C.-W. Shu, A high order discontinuous Galerkin method for $2 D$ incompressible flows, J. Comput. Phys. 160 (2000), 577-596. MR 2000m:76079

22. — A numerical example on the performance of high-order discontinuous Galerkin method for 2D incompressible flows, Discontinuous Galerkin Methods: Theory, Computation and Applications, Lect. Notes Comput. Sci. Eng., vol. 11, Springer, 2000, pp. 369-374.

23. J.-G. Liu and Z.-P. Xin, Convergence of a Galerkin method for $2 D$ discontinuous Euler flows, Comm. Pure Appl. Math. 53 (2000), 786-798. MR 2000m:76028

24. J.T. Oden, I. Babuška, and C.E. Baumann, A discontinuous hp-finite element method for diffusion problems, J. Comput. Phys. 146 (1998), 491-519. MR 99m:65173

25. T.J. Oden and C.E. Baumann, A conservative DGM for convection-diffusion and NavierStokes problems, Discontinuous Galerkin Methods: Theory, Computation and Applications (B. Cockburn, G.E. Karniadakis, and C.-W. Shu, eds.), Lect. Notes Comput. Sci. Eng., vol. 11, Springer, 2000, pp. 179-196. MR 2002d:65128

26. W.H. Reed and T.R. Hill, Triangular mesh methods for the neutron transport equation, Tech. Report Tech. Report LA-UR-73-479, Los Alamos Scientific Laboratory, 1973.

27. R. Témam, Navier-Stokes equations. Theory and numerical analysis, North-Holland, 1979. MR 82b:35133

28. A. Toselli, hp-discontinuous Galerkin approximations for the Stokes problem, Tech. Report 2002-02, Seminar for Applied Mathematics, ETH Zürich, 2002, to appear in Math. Models Methods Appl. Sci.

School of Mathematics, University of Minnesota, Vincent Hall, Minneapolis, MinNESOTA 55455

E-mail address: cockburn@math.umn.edu

Institut für Angewandte Mathematik, Universität Heidelberg, Im Neuenheimer Feld 293/294, 69120 Heidelberg, Germany

E-mail address: kanschat@dgfem.org

Department of Mathematics, University of Basel, Rheinsprung 21, 4051 Basel, SWITZERLAND

E-mail address: schotzau@math.unibas.ch 